\title{
Physiology of heartbeat reversal in adult Drosophila melanogaster (Diptera: Drosophilidae)
}

\author{
KAREL SLÁMA \\ Institute of Entomology, Biological Centre of Czech Academy of Sciences, Drnovská 507, 16100 Praha 6, Czech Republic; \\ e-mail: slama@entu.cas.cz
}

Key words. Peristaltic heartbeat, synchronic heartbeat, pulse-light optocardiography, thermocardiography, strain-gauge cardiography

\begin{abstract}
Heartbeat patterns were monitored in the living bodies of decapitated adult flies using several electrocardiographic methods (pulse-light optocardiography, thermocardiography, strain-gauge cardiography). Unlike other insect species, in which there is a peristaltic segmental propagation of cardiac contractions, Drosophila uses extremely efficient synchronic cardiac contractions. The rate of synchronic cardiac pulsation, which is characterized by simultaneous propagation of anterograde systolic contractions along all the segments of the heart, is relatively fast $(\sim 4 \mathrm{~Hz}$ at room temperature). This pulsation is used mainly for the vigorous pumping of haemolymph into the head and thorax through a narrow elastic tube, the aorta (anterograde I heartbeat). In addition, this synchronic pulsation is also used to enhance the circulation of haemolymph throughout the abdominal body cavity (anterograde II heartbeat). The switch between thoracic (anterograde I) and abdominal (anterograde II) haemolymph circulation is regulated by periodically alternating, tetanic contractions and relaxations of the conical heart chamber (ventricle). In the latter there is a pair of slitlike apertures, which are closed or opened by contraction or relaxation of the organ, respectively. During contraction of the conical chamber, the apertures are tightly constricted for several seconds and haemolymph is pumped forwards into the aorta (anterograde I heartbeat). Conversely, during relaxation of the conical chamber, the apertures are wide open for a few seconds, haemolymph leaves the heart and leaks out through open apertures and circulates from the tail to the base of the abdomen. The backward oriented, retrograde heartbeat recorded in other insects, has a lower frequency (1 to $2 \mathrm{~Hz}$ ), occurs in Drosophila only sporadically and usually in the form of individual or twinned systolic peaks of large amplitude. Unlike the synchronic nature of the anterograde I and II cardiac contractions, propagation of the relatively slow retrograde heartbeat is by peristalsis. The newly discovered, compact ventricle with atrium and synchronic functioning of the insect heart shows structural and functional analogies with the functioning of the human heart.
\end{abstract}

\section{INTRODUCTION}

Pulsations of the dorsal vessel in insects with a transparent cuticle were first observed a long time ago. In 1669, Malpighi described the basic features of the insect heartbeat, including reversal of the peristaltic waves and the myogenic nature of the action potentials of the heart (quoted by Jones, 1977). Unfortunately, we still have a poor understanding of the physiological regulation of insect heartbeat (reviews by Jones, 1977; Miller, 1997). This is because most cardiological studies are done on hearts explanted into saline in vitro (review by Miller, 1985). These explanted hearts or "semi intact" preparations of hearts in saline are removed from their physiologically important feed-back substrate, the haemolymph. Such hearts beat for some time, however, the contractions are indifferently uniform and show certain pathophysiological symptoms.

The results of in vitro cardiac experiments, indicating only an increase or decrease in heartbeat rate, have contributed little to cardiac physiology. In 1952, for example, Krijgsman used insect hearts in vitro for extensive testing of the potential cardioactive effects of a large number of chemical compounds. In some cases, he found they increased the rate of heartbeat, as expected, but dozens of other compounds that are cardioactive in vertebrates gave inconclusive results. Since then there have been numerous in vitro assays of insect heart preparations using cardiostimulatory and cardioinhibitory compounds (reviews by Miller, 1979, 1985, 1997; Bodmer et al., 2005). The difficulty of studying the physiological regulation of the heartbeat in the living insects is increased by the serious disturbances caused by locomotion and, especially, by extracardiac pulsations in haemocoelic pressure. These pulsations often have a similar or higher frequency than the heartbeat and more frustratingly up to a 500-fold stronger effect on changes in haemocoelic pressure (Sláma, 2008).

Data on the physiology of the insect heartbeat can be found in an early textbook by Wigglesworth (1965) and review articles by Richter (1973), Jones (1977) and Miller $(1985,1997)$. The current textbooks on insect physiology (Nation, 2002; Klowden, 2007) contain only a few general facts. There are certain well substantiated indications that the insect heart has some common morphogenetic characteristics with the human heart. According to Akasaka et al. (2006), of particular interest to cardiac studies is Tinman (Tin), an NK homeodomaincontaining transcriptional factor that is the earliest cardiac lineage cell marker and is necessary for the early specification of the primordial heart. It plays an important role in the determination of heart development in both Droso- 
phila and humans (Bodmer et al., 2005; Occor et al., 2007; Zeitouni et al., 2007 for review). The hearts of both these phylogenetically distant organisms work exclusively on the basis of a purely myogenic, involuntary principle; there are no cholinergic neuromotoric synapses within the myocardium. Moreover, the insect heart contains a pacemaker regulatory nodus in the terminal heart ampoule (Sláma, 2006), which is analogous to the atrioventricular regulatory nodus of the human heart (review by Bodmer et al., 2005).

The basic, elementary physiological function of the insect heart is the forward oriented, anterograde peristaltic pulsation. The larval stages of most endopterygote insects exhibit no heartbeat reversal, the stereotypic and usually continuous anterograde heartbeat representing the basic function of the heart.

During and after metamorphosis, during the development of extensive thoracic musculature and formation of the narrow aortan tube, the anterograde heartbeat (mainly thoracic circulation) is periodically supplemented by the reciprocal, purely abdominal, backward oriented or retrograde cardiac pulsation. This striking feature of the insect circulatory system is generally known as heartbeat reversal. It is composed of periodic alternations of three, physiologically distinct phases: 1. a forward oriented, anterograde heartbeat; 2 . a stationary period or diastasis, and; 3. a period of backward oriented, retrograde peristalsis. The principal function of anterograde heartbeat is to pump haemolymph into the head and thorax. This task is usually accompanied by localized bouts, or a consolidated series of faster systolic contractions. The purely abdominal, retrograde heartbeat (cf. Sláma, 2006) circulates haemolymph from the base to the end of the abdomen. The rate of retrograde heartbeat is always slower, usually half that of the anterograde rate. In certain cases (diapausing pupae) there are individual retrograde systolic contractions of large amplitude or a small series of 2 to 6 pulses separated by longer stationary periods (Sláma, 2006, 2008).

In Drosophila, the anatomical structure and development of the heart was intensively studied and reviewed by Rizki (1978). He describes the formation of the anterior conical heart chamber during metamorphosis and predicts, quite prophetically, that the origin of the conical chamber might be responsible for reversal of the heartbeat. Currently there are more detailed anatomical descriptions of the dorsal vessel in Drosophila (Curtis et al., 1999; Dulcis \& Levine, 2005; Wasserthal, 2007; Zeitouni et al., 2007).

Visual observations on the changes in the heartbeat rate of insects were first made a long time ago (reviews by Wigglesworth, 1965; Jones, 1977). In Drosophila, Nichols (1999), Zornik and co-workers (1999) investigated in vivo effects of "cardioactive" peptides using optical methods. Unfortunately, the results were expressed only as percentage increase in heartbeat rate 3 min after the injection. They did not notice heartbeat reversal or extracardiac pulsations. The cardiac pulsations observed in vitro were intensively investigated by
Bodmer and his co-workers (Bodmer, 1995; Bodmer \& Venkatesh, 1998; Ocorr et al., 2007; for review see Bodmer et al., 2005). The aim of their studies was to determine the effects of ageing on the circulatory performance of the dorsal vessel. They found differences in the rate of heartbeat between young and very old flies. In addition, they give a good account of the genetical factors involved in the embryonic formation of the dorsal vessel and found structural and developmental analogies between the hearts of Drosophila and those of vertebrates (review by Bodmer et al., 2005).

There were several optoelectronic studies published on the heartbeat of Drosophila in 2005, which include data on heartbeat reversal. Dulcis \& Levine (2005) used a high speed video technique to record the heartbeat in semi-intact preparations (amputated legs). They observed periodical changes in optical density alternating over intervals of several seconds. These changes were interpreted as the systole and diastole of the heart, with much faster pulsations (approximately $3.5 \mathrm{~Hz}$ ) indicated as minisystoles and minidiastoles. The forward oriented, anterograde heartbeat was considered to be faster than the retrograde one. Sláma \& Farkaš (2005) investigated the heartbeat during the whole postembryonic development of Drosophila, using a touch-free, multichannel recording technique based on the use of visible pulsed-light. They found that the smallest larvae of the first instar exhibited the fastest heartbeat rates ever recorded $(7 \mathrm{~Hz})$. This fast larval heartbeat was exclusively anterograde. During larval growth, the frequency of anterograde pulsations successively decreased to some $2.6 \mathrm{~Hz}$ during and after puparium formation, with progressively prolonged periods of cardiac rest. Heartbeat reversal was first noticed during metamorphosis, in a two-day-old puparium, coincident with the formation of the conical heart chamber. Similarly as in other insect species and stages, the anterograde heartbeat of pupal or pharate adults of Drosophila occurred in localized bouts of systolic contractions. The rate of the anterograde heartbeat was always more than twice that of the retrograde heartbeat. Recently, Wasserthal (2007) used infra-red radiation and linear micro chip receivers to obtain prolonged recordings of the heartbeat of intact adults of two species of Drosophila. He found regular switches between anterograde and retrograde heartbeats at intervals of several seconds. In contrast to all other reports (review by Wigglesworth, 1965; Johnson, 1977; Miller, 1997; Sláma, 2006), the rate of anterograde heartbeat was claimed to be slower than the retrograde rate (Wasserthal, 2007).

In this work I have reinvestigated the heartbeat patterns in adult Drosophila, using immobile, decapitated flies. These flies remain motionless, but retain autonomic regulation of essential physiological functions. The results of optoelectronic recordings were compared with the data obtained by other electrocardiographic methods based on substantially different physical principles (thermography, strain-gauge posimetric recording). Due to the small size of Drosophila, it was necessary to develop extremely sen- 
sitive, nanotechnological micromethods, in order to discern and analyse the individual strokes of the heart.

\section{MATERIAL AND METHODS}

The recordings were made on adult males and females of wild type Drosophila melanogaster, Oregon R strain. Investigations were mostly made on decapitated young adults (2- to 6-days-old). Before decapitation, the flies were immobilized by immersing them in water for 5 to $10 \mathrm{~min}$. The head of the immobilized flies was carefully separated from the rest of the body using microscissors. The wound was blotted and covered immediately with a small droplet of melted wax, which was delivered by a gently heated platinum wire loop of a miniature electrical thermocautery apparatus. A droplet of melted wax was further used for fixing the decapitated fly to a small plastic plate, with its legs stretched out. The decapitated flies remained motionless and lived for many hours or even a few days, when kept under humid conditions. The autonomic physiological functions (respiratory, circulatory, digestive, excretory) recovered within $10 \mathrm{~min}$ and remained in a fully functional state in the decapitated flies for many hours. The autonomic functions are controlled in all insects from the mesothoracic ganglion of the ventral nerve cord by a brain-independent, neuroendocrine system known as the coelopulse system (Sláma, 2000, 2006, 2008). The headless adults retain the forward oriented, anterograde heartbeat, but as the circulated haemolymph cannot reach the head, it leaves via excurrent ostia in the thoracic aorta.

Neurotoxic materials (narcotics, insecticides, trimethylamine), which are often used for chemical immobilization of flies, were not used. Neurotoxic compounds do not inhibit heartbeat, which is myogenic, but abolish all cholinergic neuromuscular functions, including extracardiac pulsations. This seriously disturbes respiration (hypoxia, hypercapnia) due to the lack of ventilatory movements. The intoxicated flies are suffocating (Coats, 1982), which sooner or later indirectly affects the heartbeat.

Electrocardiographic methods used in this study on Drosophila were previously described by Sláma $(2000,2003,2006)$ and used for recording heartbeat of relatively large insects (pupae of Manduca). The use of transmitted or reflected pulsedlight in optoelectronic recordings of heartbeat in Drosophila was previously described by Sláma \& Farkaš (2005). The advantage of using pulsed-light is that it reduces electrical interference between many sensors packed in a small area. Each pulse-light channel uses a special, electronically filtered frequency (Sláma, 2003, 2006). In this study the noise-to-signal ratio was maximally amplified using high resolution nanotechnology. For thermographic or posimetric recordings perfectly stabilized $5 \mathrm{kHz}$ oscillators were used to supply alternating current to the strain-gauges and thermographic bridges. The sensors were integrated using a multichannel, electronically filtered, M-1000 tensiometric unit (Mikrotechna, Czech Republic) (for more details see Sláma, 2000, 2003). The strain-gauge transducers were connected to the abdomen of Drosophila by thin metallic wires (O. D. $50 \mu \mathrm{m})$. Thermographic recordings of such small insects were achieved by using the smallest available sensors $(250 \times 350 \mu \mathrm{m})$. The $5 \mathrm{kHz}$ supply to the AC circuitry (options 1, 2, $4 \mathrm{~V}$ ) was adjusted to temperature gradients around the sensors of not more than $2^{\circ} \mathrm{C}$ above the body temperature of the fly. The strain-gauge posimetric sensors used to measure the heartbeat (Sláma, 2000) were also adjusted to record movements over a subnanometer range. This was achieved by protecting the fly-sensor setup with a heavy metallic box, which buffered interference caused by sudden movements and changes in the temperature of the surrounding air.
These electrocardiographic methods were used to record the heartbeat of Drosophila over periods of at least 2 to $4 \mathrm{~h}$. The heartbeat of a limited number of the flies were also continuously recorded overnight. The heartbeat of 83 decapitated adult flies were recorded. The recordings were made 2 to $5 \mathrm{~h}$ after decapitation, in order to avoid the effect of the initial stress caused by the treatment and later inconsistencies resulting mostly from changes in humidity. Under humid conditions, however, the decapitated flies survived for at least one day. Where necessary, the numbers $(n)$ of flies in each experimental group is cited in the text. The final, multiplied and decoded DC voltage output signals from the sensors, retrieved from the M-1000 unit, were recorded on a PC, using a 4-channel data acquisition and analysis system DI-158U purchased from DATAQ instruments (Ohio, USA). Since there is no such thing as an average fly, the results were evaluated by carefully inspecting and comparing the recordings. In order to illustrate the text, recordings of more or less representative traces were selected for presentation in the Figures.

\section{RESULTS}

\section{Functional morphology of the heart}

As in all other endopterygote insects, the dorsal vessel in adult $D$. melanogaster consists of two parts. A narrow thoracic tube, which is conventionally termed the thoracic aorta, and an abdominal heart with all the essential features of a cardiac circulatory organ. The heart of intact flies is transparent and almost invisible in the transparent haemolymph. The most important structural and functional part of the heart is the conical chamber (ventricle), which is in the proximal part of the heart, located at the base of the abdomen ( $\mathrm{A}_{1}$ abdominal segment) between two large tracheal sacs. The front of the conical chamber is attached to abdominal wall by ligaments. On ventral side it is connected to a narrow tube, the aorta, which leads ventrally into the central thoracic cavity, which is surrounded by extensive musculature and contains the intestine. The conical chamber has a pair of apertures on each side of the aorta. The apertures open into the venous channels between the thorax and abdomen or, alternatively, they close tightly for several seconds when the conical chamber contracts. In contrast to the 4 pairs of passively operated incurrent ostia, the slit-like apertures in the conical chamber are actively operated, bidirectional valves. During the vigorous, anterograde pumping of haemolymph into the head and thorax, the conical chamber contracts and the apertures are closed. Whereas, during the purely abdominal, anterograde II cardiac pulsation, the conical chamber expands, the appertures are open and haemolymph flows out into the haemocoelic cavity at the base of the abdomen.

In the posterior part of the conical chamber there is a pair of normal incurrent ostial valves. The conical chamber is connected to a round or pear-shaped atrium, which acts as a reservoir for haemolymph during vigorous anterograde pulsations, especially in gravid females. Physiological function of the atrium is similar to atrial functions in the human heart. The distribution of the dark stained pericardial cells in Fig. 1 help in identifying the contours of the conical heart chamber and the atrium. The distal part of the heart ends in a terminal chamber 


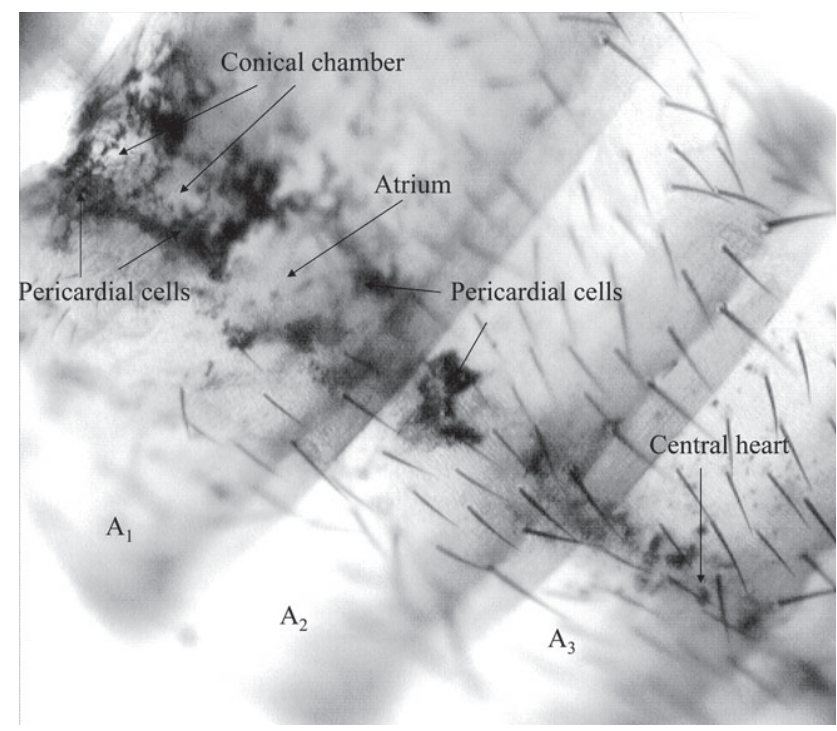

Fig. 1. Photograph showing the contours of the heart visible through transparent tergites of the 1st to 3rd abdominal segments of an adult female of D. melanogaster. The contours of the heart are outlinned by the darkly stained pericardial cells.

(terminal ampoule), which is characterized by a thick myocardium wall and increased number of adjacent pericardial cells. At the distal end of the heart there is a valvelike orifice, which remains open during the backward retrograde pulse.

Fig. 1 shows that between the conical ventricle at the front and terminal chamber at the rear of the heart, there is a narrow, transparent central part. This is composed of 3 metameric myocardium segments with 3 pairs of incurrent ostial valves. The ostia are one-way, passive mechanical valves, which enable the haemolymph to flow from the pericardial sinus into the lumen of the heart. The flow of haemolymph through the incurrent ostia is restricted to short, intermittent intervals of lowered diastolic, intracardiac pressure. From the mechanical point of view, the dorsal vessel of adult Drosophila is an elastic, tubular organ, freely suspended in the pericardial sinus of the haemocoelic cavity. The length of the dorsal vessel can be substantially increased or contracted during the large, longitudinal movements of the abdomen. In an intact fly, the heart is transparent and almost invisible. Fig. 1 shows, however, that the myocardium wall of the heart can be visualized and used for optical recording by injecting black india ink into the haemocoele. The ink particles are absorbed by the pericardial cells, which become dark stained and enhance optoelectronic recording of the heart movements.

\section{Cardiac versus extracardiac haemocoelic pulsations}

The dorsal vessel of insects is a relatively weak circulatory organ, which moves haemolymph between different compartments of the open haemocoelic cavity, but generally it is not constructed for pumping haemolymph against any larger barrier of mechanical pressure. Due to this, insects have evolved all kinds of auxilliary extracardiac circulatory adaptations that are of both neurogenic and a purely myogenic nature. For example, there are strong extracardiac pulsations in haemocoelic pressure, which are produced by rhythmic contractions of the intersegmental abdominal muscles. These purely neurogenic pulsations have 50- to 500-fold greater effect on changes in haemocoelic (internal) pressure than the heartbeat. In addition, there are other extracardiac pulsations that are of a myogenic nature. These are essentially pulsations of the ventral diaphragm, peristaltic movements of the intestine and cephalic or scutellar accessory pulsatile organs. They enhance unidirectional haemolymph circulation through the appendages or betwen the cephalic, thoracic and abdominal body compartments.

The strong extracardiac pulsations can be easily confused with the heartbeat, although there exist several physiological criteria for their distinction: (a) Extracardiac haemocoelic pulsations are neurogenic in contrast to the myogenic heartbeat; (b) Propagation of extracardiac pulsations is systemic, while the heartbeat is usually peristaltic, and; (c) Extracardiac pulsations produce mainly a tidal movement of haemolymph, while heartbeats circulate the haemolymph. The accessory extracardiac circulatory functions involve, for example, large contractions of abdominal segments (abdominal pressure pump), which cause tidal reflushing or washing of tissue and organs with haemolymph. The large abdominal contractions can also induce a massive tidal flow of haemolymph between the thoracic and abdominal haemocoelic cavities. These tidal currents are apparently not sufficient for restraining local occlusions or clogging of haemolymph, which is executed by accessory pulsatile organs that help to circulate haemolymph in those parts the heartbeat induced circulation does not reach. The danger of tidal, stationary haemolymph occlusions are especially acute in gravid females of Drosophila, which contain densely packed internal organs with extremely high metabolic demands. Finally, the body contains a number of different tissue outgrowths, simple membranes or membranaceous septa, which function as passive, one way valves, directing a stream of haemolymph in response to large peaks in haemocoelic pressure.

Fig. 2 shows extracardiac pulsations recorded by electronic sensors placed on the end of the telescopic abdomen (see insets). The best responses of this type are obtained when the sensor is on the ventral side of the last abdominal segment. Depending on the age and physiological state of the flies before decapitation, the pulsation was continuous or occurred in a shorter or longer series separated occasionally by longer periods of rest. The rate of these pulsations was close to $1 \mathrm{~Hz}$ (=60 per min., at room temperature). This pulsation seriously interferes with the backward oriented, retrograde heartbeat ( 1 to 2 $\mathrm{Hz}$, see below), which can be recorded independently from the opposite dorsal side, just above the terminal chamber of the heart. Fig. 2A shows the periodically repeated bouts of the ventral extracardiac pulsations recorded by the strain-gauge method. The movement of the last abdominal segment associated with these pulsations (Fig. 2A) is relatively small, only some 10 to $50 \mathrm{~nm}$. 


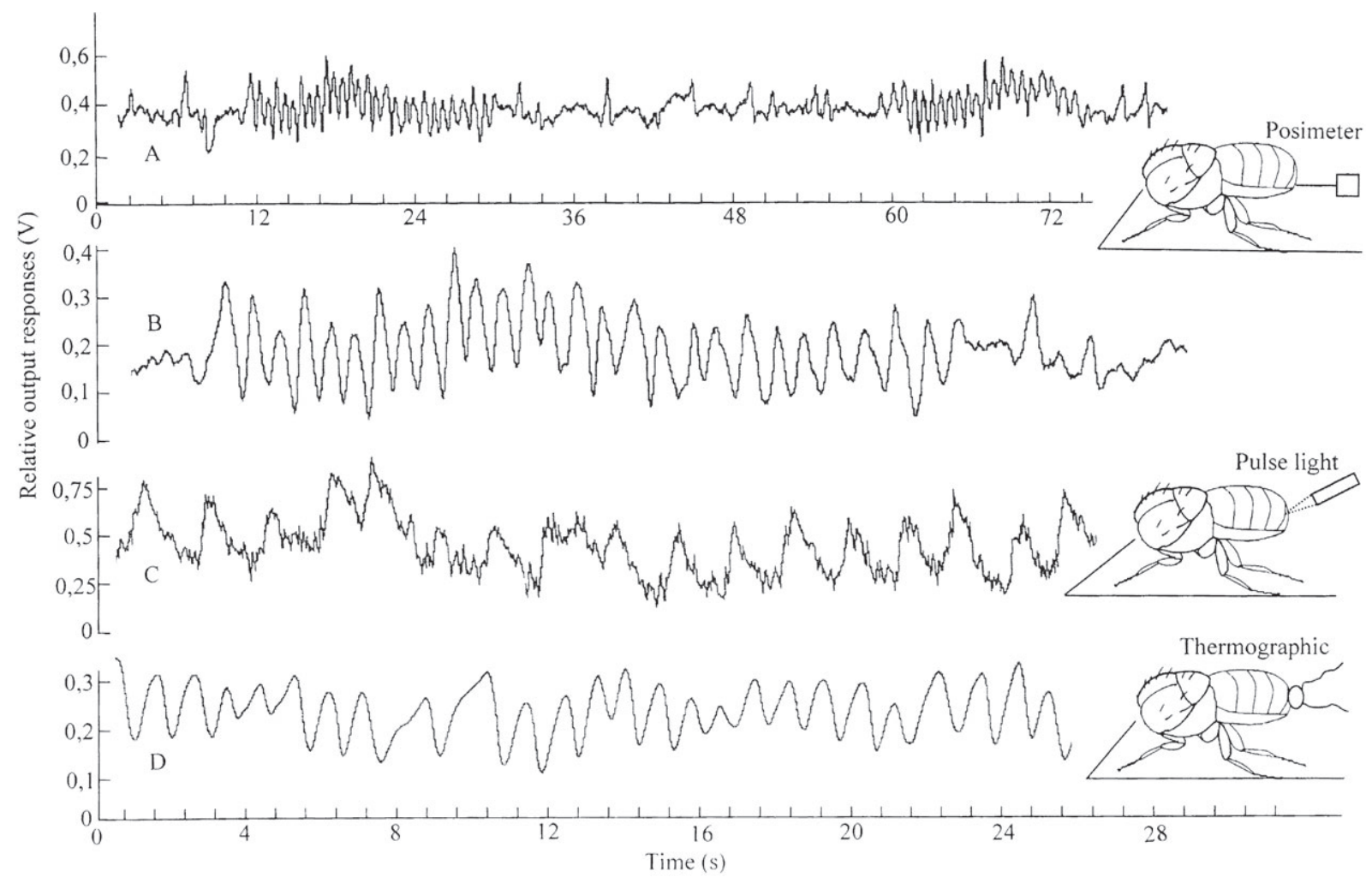

Fig. 2. Extracardiac haemocoelic pulsations of the ventral perineural sinus, recorded using several eletrocardiographic methods (A - posimetric, B - optoelectronic and C - thermographic; see insets). The sensors were attached to the ventral side of the end of the abdomen of young females of D. melanogaster, from 2 to $5 \mathrm{~h}$ after decapitation $\left(25^{\circ} \mathrm{C}\right)$.

It is usually camouflaged by substantially larger segmental movements exhibited during extracardiac pulsations in haemocoelic pressure. The traces B $+\mathrm{C}$ and $\mathrm{D}$ in Fig. 2 show the patterns of the above described pulsations when recorded by pulse-light and thermographic sensors (insets in Fig. 2).

The above described $1 \mathrm{~Hz}$ extracardiac pulsations were recorded on the ventral side of the abdomen, over the whole length of the ventral sinus of decapitated adults of Drosophila (see Fig. 3A). It can be reasonably assumed that these regular extracardiac pulsations were made by peristaltic movements of the crop or ventral diaphragm. Occasionally, the pulsations were coupled with large peaks of lower frequency (see Fig. 3B). According to similar features found in other insects and also in Drosophila (see Sláma \& Farkaš, 2005), these low frequency pulses could be ascribed to slow peristaltic contractions of the gut. The fused, ventral nerve cord of adult Drosophila has increased demands for metabolites, but the circulatory exchange does not reach all parts of the abdomen. In addition, the ventral nerve cord is enclosed within the narrow ventral thoracic sinus and thus practically out of the reach of the heart. Circulatory functions in this area are facilitated by $1 \mathrm{~Hz}$ myogenic pulsations of other visceral organs, oesophagus, crop ducts or ventral diaphragm.

Important circulatory and respiratory adaptations of neurogenic origin are represented in insects by extracardiac pulsations in haemocoelic pressure. These pressure pulses (abdominal pressure pump) are produced by tele- scopic contractions or relaxations of intersegmental abdominal muscles. Nerve impulses of the autonomic neuroendocrine system (coelopulse) originate in thoracic ganglia of the ventral nerve cord (for references see discussion). In many insect species, the frequency of extracardiac haemocoelic pulsations is similar or higher than the heartbeat. Fig. 4A shows, however, that the strong extracardiac haemocoelic pulsations in Drosophila are much slower (only about 11-12 contractions per min) than the heartbeat ( $4 \mathrm{~Hz}=240$ strokes per min). Young males or starved females before decapitation exhibited large, individual contractions (expirations of air) occurring regularly with a periodicity of several minutes (Fig. 4, lower record).

The extracardiac abdominal contractions shown in Fig. 4A and 4B apparently cause positive peaks in haemocoelic pressure, affecting the inflation or deflation of large tracheal sacs present at the base of the abdomen. The sacs occur on each side of the conical heart chamber and have both respiratory and circulatory functions. The peaks in abdominal contractions (increased internal pressure; see Fig. 4A) are obviously associated with discharge of gas through one or more spiracles that are just open. In this way Drosophila, like some other insects, can actively regulate spiracular breathing. Occasionally, I observed extracardiac pulsations with peaks of reversed polarity, i.e. peaks associated with sudden abdominal prolongation, not contraction (see Fig. 5). Based on comparisons with similar peaks found in other insects, we can reasonably 


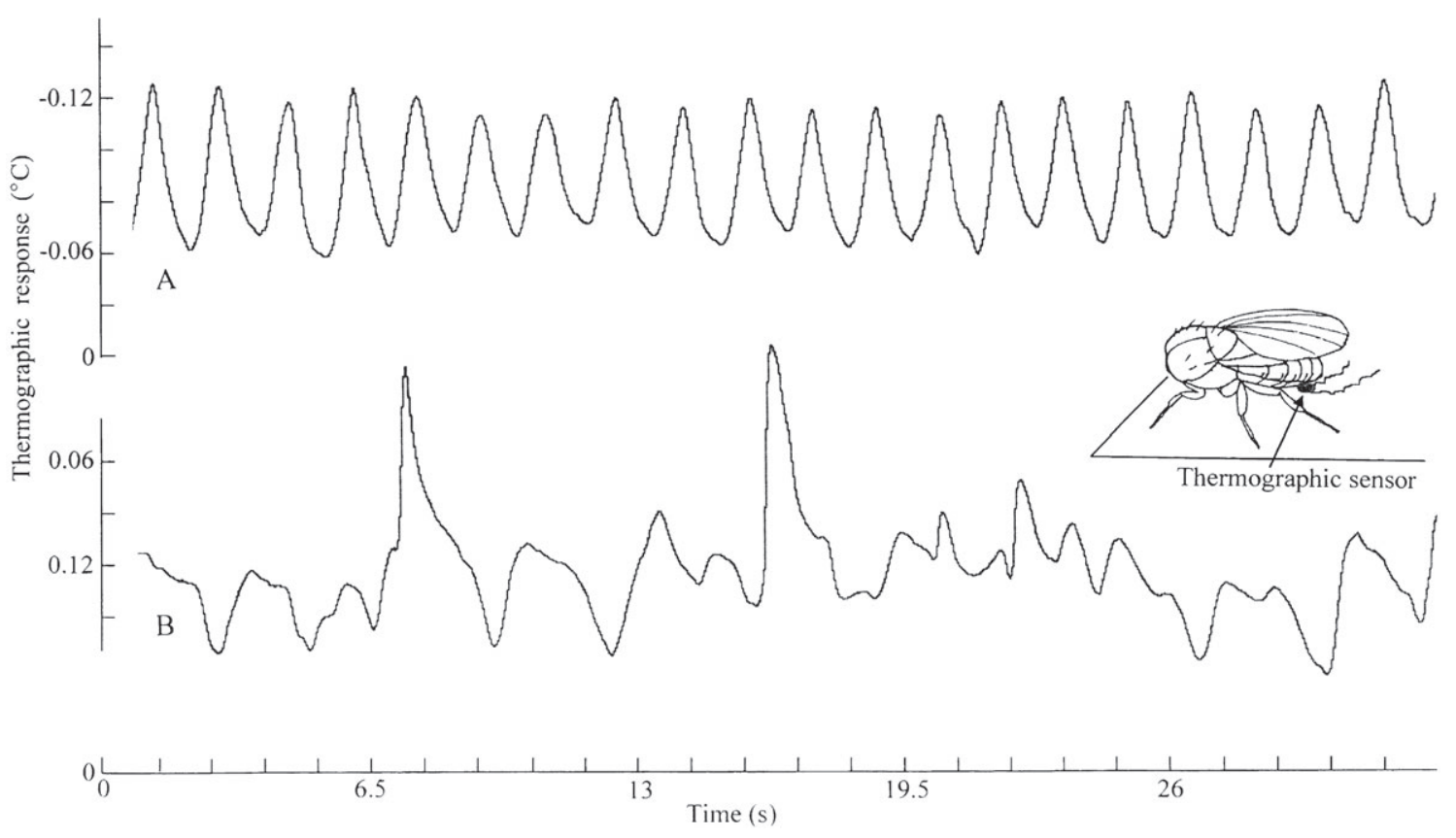

Fig. 3. Extracardiac pulsations of the ventral perineural sinus (crop or ventral diaphragm?) recorded by thermographic sensors attached to the middle of the $3 \mathrm{rd}$ abdominal sternite (inset) of 5-day-old females of D. melanogaster, 2-5 h after decapitation.

assume that these reciprocal peaks are associated with the physiologically regulated intermittent inspirations of air.

Inhibition of extracardiac pulsations by neurotoxic compounds (narcotics, insecticides, trimethylamine), which are often used for the chemical immobilization of flies during heartbeat measurements, seriously disturbs the physiological balance of the investigated specimens (Coats, 1982). The chemically inflicted abolition of the cholinergic extracardiac pulsations leads to respiratory acidaemia, hypoxia, hypercapnia and finally, to suffocation and death. The cessation of neuromuscular functions does not stop the myogenic heartbeat, however, it disturbs

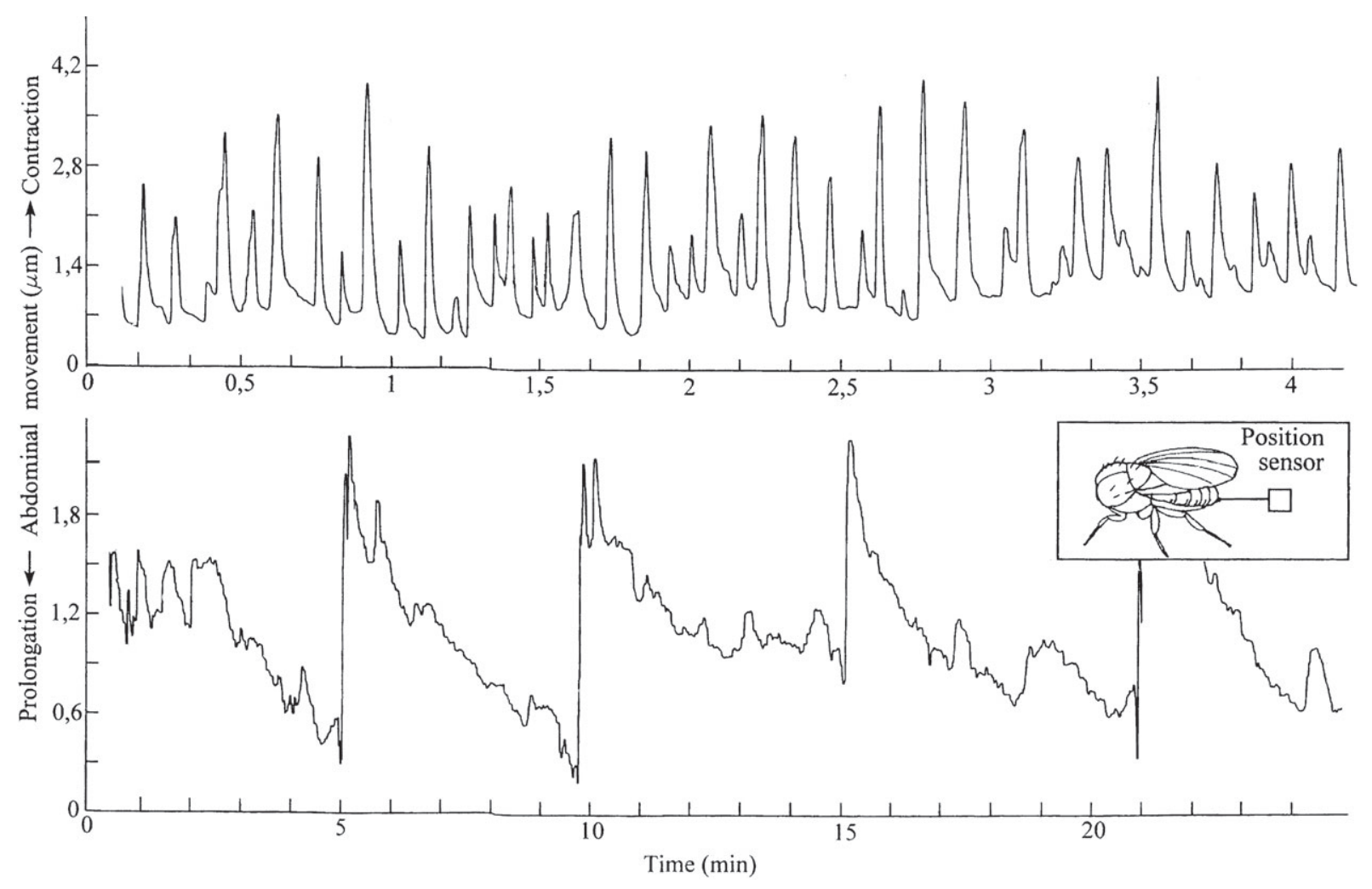

Fig. 4. Large extracardiac pulsations caused by longitudinal contractions of intersegmental abdominal muscles (8-12 per min), recorded by a sensor on the end of the abdomen (inset) of a decapitated gravid female. Lower trace shows a similar periodically repeated abdominal contraction of one every $5 \mathrm{~min}$ recorded from a young decapitated adult male of $D$. melanogaster, at $25^{\circ} \mathrm{C}$. 


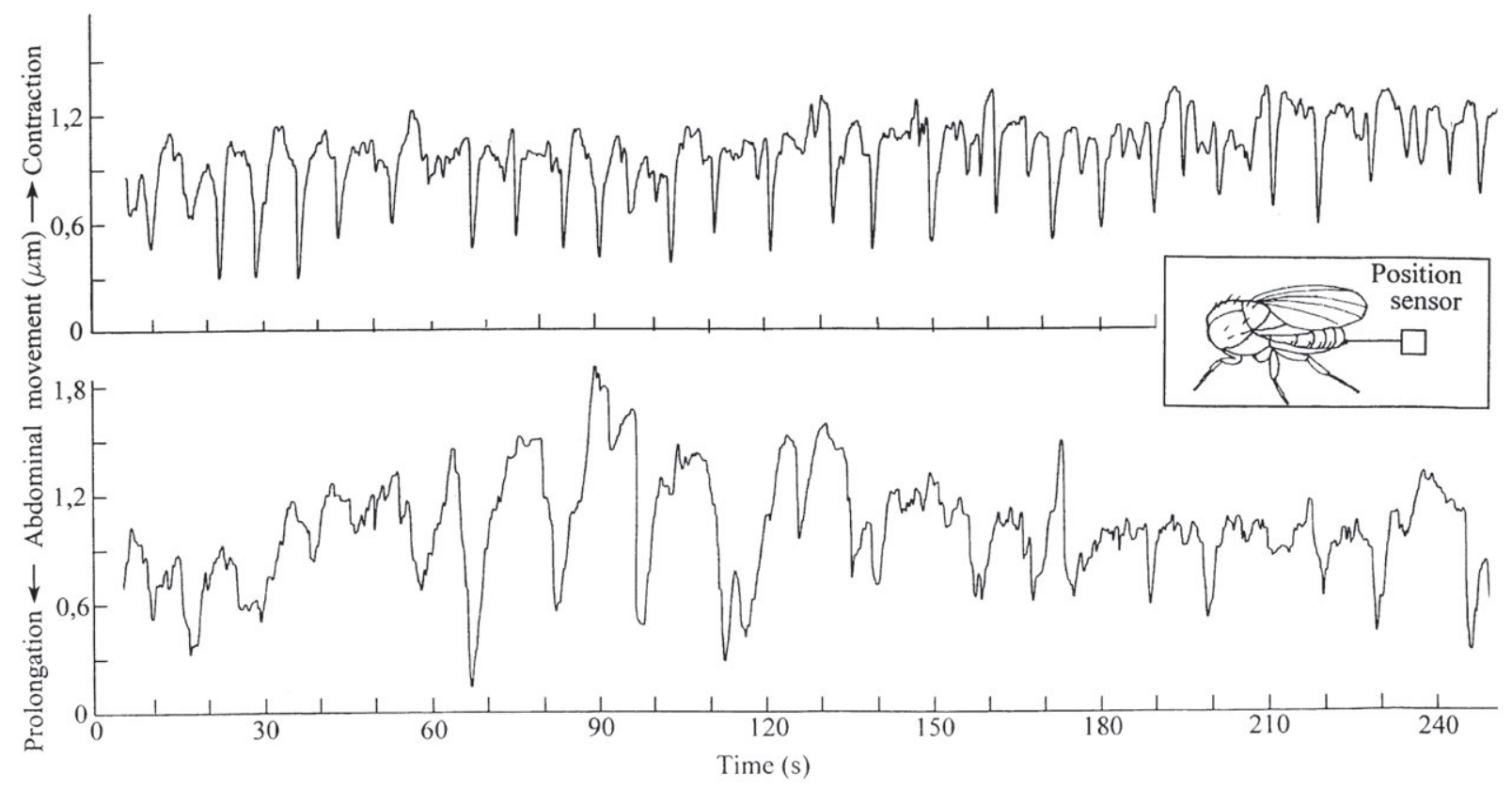

Fig. 5. Large extracardiac peaks of abdominal extension associated with inspirations of air through spiracles. Upper record is of regular pulses with a periodicity of 5-6 per min, lower one of less regular pulsations. Young decapitated females of $D$. melanogaster, recorded at $25^{\circ} \mathrm{C}$.

the endogenous metabolic balance in the body so much that it consequently affects the heartbeat patterns in unpredictable indirect ways. In order to avoid the use of neurotoxic chemicals, we narcotized the flies by a few minutes of submersion in water and then immobilised them by decapitation. The headless flies performed autonomic (brain independent) regulation of physiological functions, including the extracardiac pulsation activities described in Figs 2 to 5. After the initial stress of decapitation, the basic physiological functions of these immobile specimens (oxygen consumption) stabilized within $15 \mathrm{~min}$ or one hour. In order to standardize the conditions for the measurements, the heartbeat was recorded between 2 and $5 \mathrm{~h}$ (exceptionally one day) after decapitation.

\section{Optocardiographic recording}

Recently acquired knowledge about the heartbeat of adult Drosophila is exclusively based on optical recording methods (see discussion). With only a few exceptions, these methods were based on semi-intact (amputated legs) or in vitro measurements, which often gave inconsistent results and interpretations. According to my experience, the main obstacle in this respect is connected with the transparent and invisible nature of haemolymph. Thus the optical recordings show only movements of the external wall of the heart, without direct relation to the direction of the internal haemolymph flow. The tubular heart of adult Drosophila is floating freely suspended in the pericardial cavity, except for the conical chamber which is fixed by the ligaments. The movements of the heart can be perhaps best exemplified by comparison with what can occur in a garden hose held by a gardener. A sudden increase in water flow within the hose, like the systolic stroke within the heart, invariably produces a regressive, kick-back response in the wall. The hose or the heart tube simply moves in the opposite direction. Neglecting this fact may cause serious confusion and misunderstanding in the identification of anterograde and retrograde heartbeats.

Fig. 6 provides an example of an optocardiographic recording made using reflection of visible pulsed-light from the pericardial region of an unrestrained, immobile, sitting fly. The pulse light was focused from a distance of $7 \mathrm{~mm}$ through the wings on to a small, $0.3 \mathrm{~mm}^{2}$, area over the conical heart chamber. However, these experiments always failed because the flies moved out of focus. Nevertheless, I obtained several dozens of such recordings, which indicated nothing more than a strange up and down change in the optical density of the conical heart chamber (Fig. 6A).

Occasionally, there were records of a slightly better resolution. In this case (Fig. 6B) changes in optical density reflected from the conical heart chamber revealed periods of pulsations with a relatively high frequency (usually 3-4 Hz), evidently associated with the heartbeat. Using optical methods alone, however, it was impossible to determine the direction of the intracardiac haemolymph flow. The anterograde and retrograde directions of the heartbeat indicated in Fig. 6B were obtained using complementary evidence derived from other sensors (see below).

After the disappointment of not being able to record from intact flies I used flies immobilized by decapitation. Headless flies will sit motionless for several hours. Previous studies on other insect species revealed that decapitated insects can breathe, exhale carbon dioxide, digest previously taken food and excrete. The record shown in Fig. 7 documents the successful use of the pulsed light 


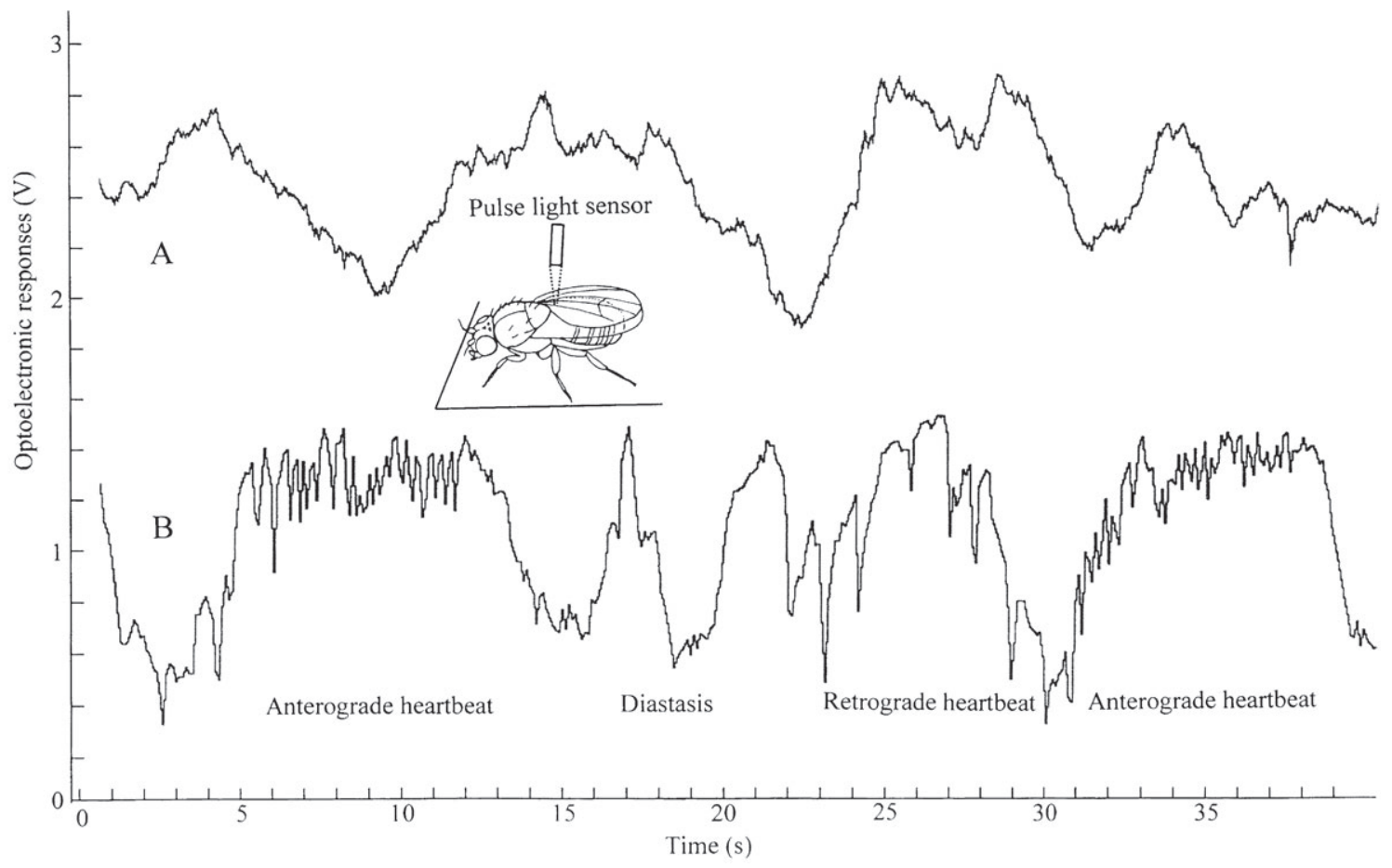

Fig. 6. Optocardiographic records obtained by visible pulsed-light, delivered by optic fibers and optically focused through transparent wings on to a small $\left(0.3 \mathrm{~mm}^{2}\right)$ integumental area above the pericardial region in the 1st abdominal tergite (inset). The reflected light, modified by movements of the heart, was collected and amplified. During the recording, the young adult male of $D$. melanogaster remained freely sitting in a Petri-dish.

technique for recording the heartbeat in decapitated Drosophila. In this case one can easily discern for the first time the different nature of the systolic contractions. For example, the bouts of fast, $4 \mathrm{~Hz}$ pulsations, evidently representing the most common anterograde heartbeat, were consistently present in all records. They were often followed by brief periods of diastasis, which is also a common cardiac feature of other insect species. During the periods of diastasis, the optical sensors revealed systolic contractions of low frequency and large amplitude (indicated by asterisks in Fig. 7), which are also common in other insects. They are a characteristic of the reciprocal, backward oriented, slower retrograde heartbeat, reported in the pupal and adult stages of many insect species (see the discussion). The record shown in Fig. 7 represents a small sample selected from 18, several hour long optocardiographic recordings of decapitated male and female adults of Drosophila.

\section{Thermographic recording}

The optoelectronic record in Fig. 7 shows certain modifications of the basic heartbeat pattern, which require further experimental clarification. In order to throw more light on these changes, it was necessary to use a high resolution, nanometer scale technique, which can record the individual systolic contractions of the heart. To this end, I used an electrocardiographic method involving contact thermography for recording from decapitated adults of Drosophila. The miniature thermographic sensors were positioned externally on the integumental surface overlying the pericardial region. A great advantage of this method is that, in addition to systolic strokes of the heart, it can also record quantitative changes in subintegumental haemolymph flow through the pericardial region. This can be briefly explained by a simple relationship: the faster the subintegumental flow of haemolymph the proportionally greater the cooling effect and electrical change recorded by the sensor. In the system used in this study, increased haemolymph flow was indicated by the recording devices by movement of the pen upwards. Conversely, arrested haemolymph flow, such as when the heartbeat ceases, can be recognised by low background values.

The above technical relationships can be practically demonstrated using the traces in Fig. 8A and 8B, which show a common thermographic heartbeat pattern recorded from a decapitated female of Drosophila. There are 4 conspicuous bouts of intensive, $4 \mathrm{~Hz}$, unidirectional heartbeats (Fig. 8A), which are characterized by increased amplitude of individual systolic contractions and, therefore, substantially increased haemolymph flow. Similar heartbeat patterns occur in pupal and adult stages of all insects. They represent the elementary physiological function of the insect heart, which is manifested by the vigorous forward pumping of haemolymph through a narrow aorta into the head. In other words, this type of thermographic record shows unequivocally the bouts of anterograde heartbeat in insects, which is indicated here as the anterograde I heartbeat.

Further inspection of the trace in Fig. 8 A shows that the bouts of intensive, anterograde I heartbeat are separated by equal or slightly longer periods of cardiac rest (low background values indicated by thermographic sen- 


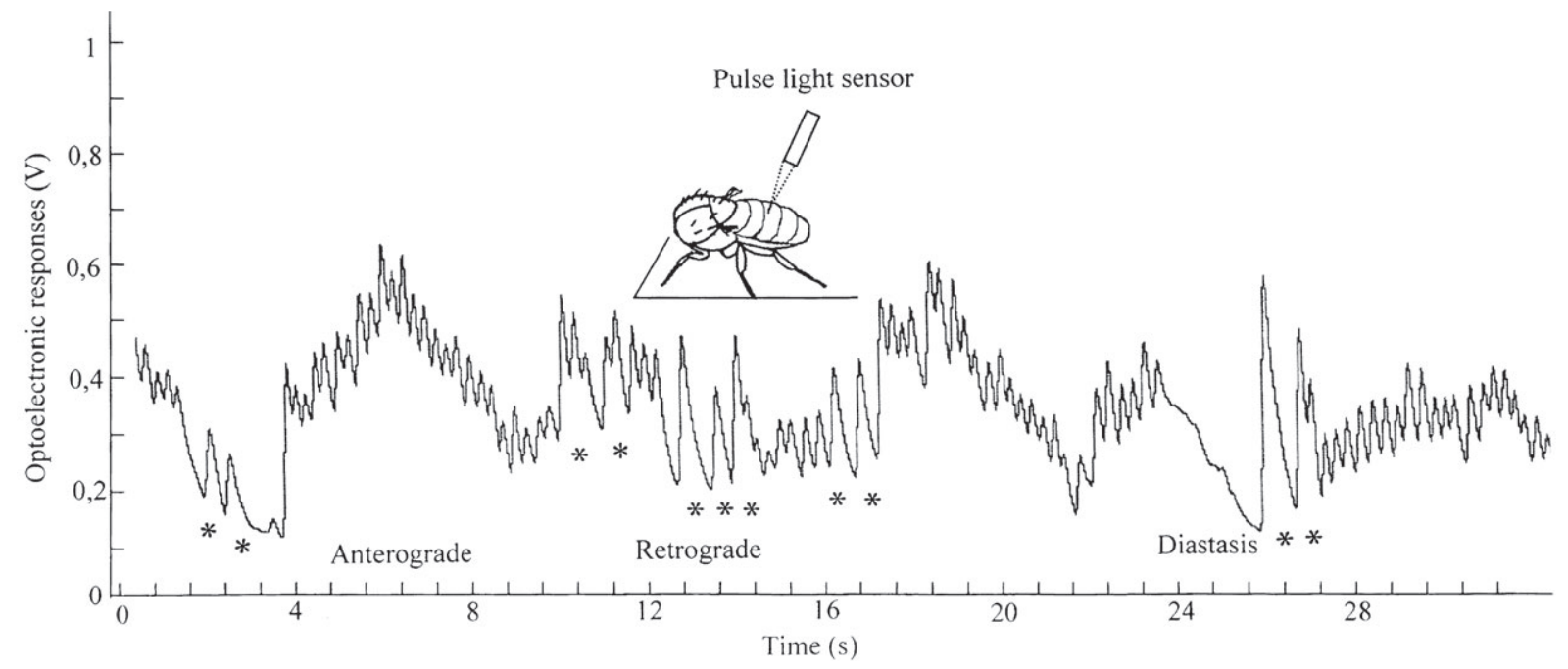

Fig. 7. Optocardiographic recording of the heartbeat using a beam of visible pulsed-light focused on to a $0.3 \mathrm{~mm}^{2}$ integumental area above the pericardial region in the 3rd abdominal tergite (inset) of a 3-day-old female of D. melanogaster, $3 \mathrm{~h}$ after decapitation, at $23^{\circ} \mathrm{C}$.

sor). It is thus obvious that these periods of diastasis can be clearly recognized by their low baseline values, which indicate simultaneously the cessation of haemolymph flow. As in most other insect species, these exclusively anterograde I bouts of heartbeat alternating with more or less regular periods of diastasis (Fig. 8A) constitute the most common heartbeat pattern and can be repeated for many hours.

It may be noted that the thermographic record shown in Fig. 8A actually displays the in vivo heartbeat pattern of adult Drosophila, demonstrating the detailed resolution of individual systolic contractions for the first time. Actually, without this sensitive measuring technique, the newly discovered modifications of the cardiac pattern would not have been found. Comparison of the traces in Fig. 8A and B shows that they are superficially similar and could thus be easily confused. The major difference, however, is that the heart in $8 \mathrm{~B}$ never relaxes. For some reason it continues to pulsate during the periods, which are normally occupied in $8 \mathrm{~A}$ by diastatic rests. The rela-

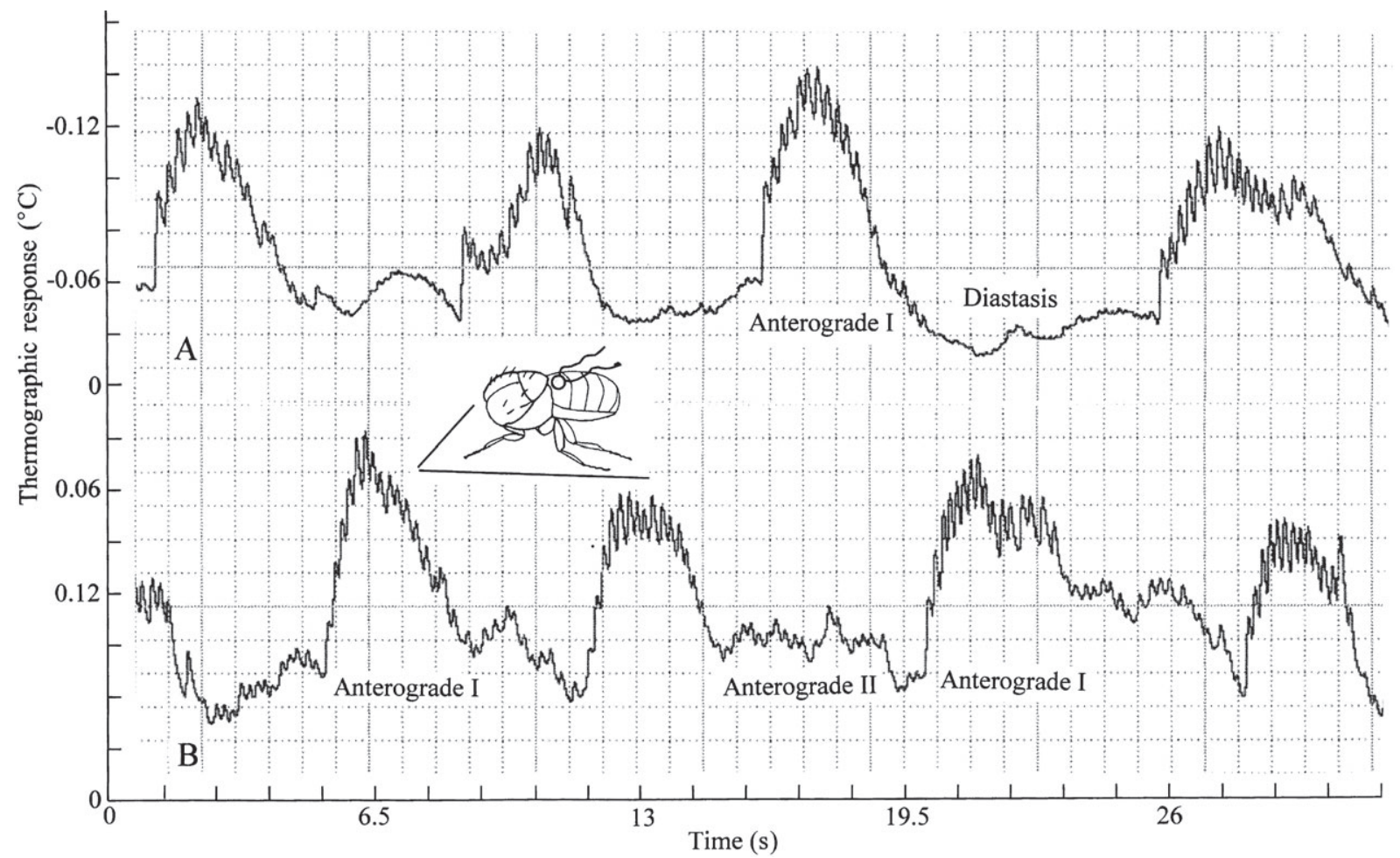

Fig. 8. Thermocardiographic records of heartbeat transduced from the pericardial region of the 1 st to 3 rd abdominal tergites. The miniature thermographic sensors were placed on the integumental surface (inset) of each of two young females of D. melanogaster. The recordings were made between the $2 \mathrm{nd}$ and 5 th $\mathrm{h}$ after decapitation, at $25^{\circ} \mathrm{C}$. 


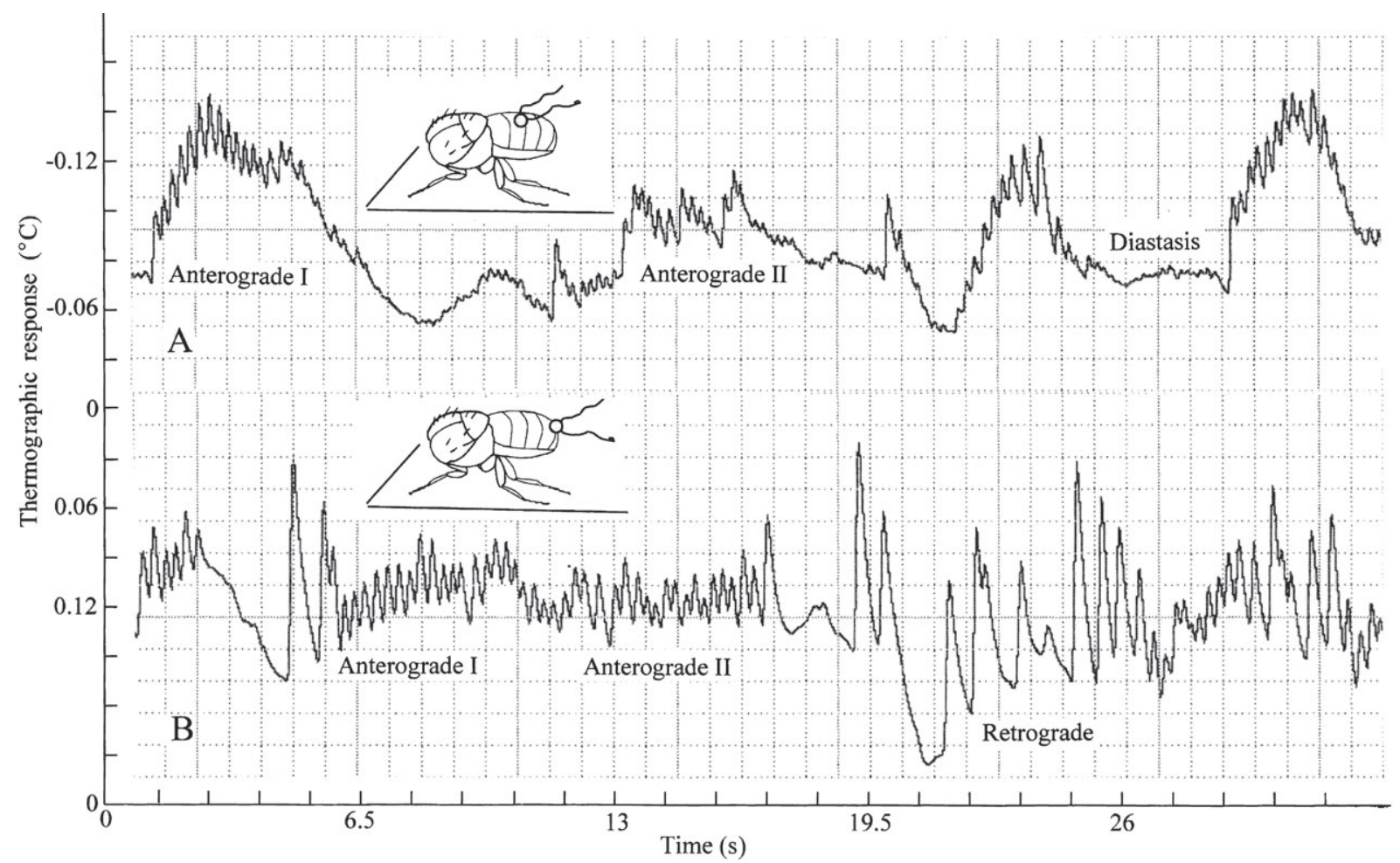

Fig. 9. Thermocardiographic records, similar to those in Fig. 8, transduced from other decapitated young females of $D$. melanogaster. For the placement of sensors in A and B see inlets.

tively small amplitude of the systolic contractions during the periods of low background levels in $8 \mathrm{~B}$ suggests a decline in intracardiac pressure. In summary: there are two physiologically distinct types of the unidirectional heartbeat, which is a newly discovered cardiological feature of Drosophila. In order to distinguish the less efficient, $3.5 \mathrm{~Hz}$ cardiac pulsation from the vigorous, $4 \mathrm{~Hz}$ anterograde I pumping of haemolymph into the head (Fig. 8B), we propose to call it an anterograde II heartbeat.

In contrast to the relatively well known changes associated with the bi-directional heartbeat reversal, little is known about the variations within the uni-directional heartbeat, represented by the anterograde I and II heartbeats recorded in Fig. 8B. The data in the literature (see discussion) automatically ascribes the phenomenon of anterograde I and II to heartbeat reversal, assuming that the first is anterograde and the second retrograde. We have ample experimental evidence, however, that anterograde II is definitely not a retrograde heartbeat. This evidence is corroborated by extensive retrospective analysis of all available, prolonged thermographic records $(n=$ 17). The strong (anterograde I) and weaker (anterograde II) cardiac pulsations depicted in Fig. 8B were based on identical systolic principles and both propagated haemolymph only anteriorly.

Occasionally, under certain physiological conditions, the anterograde I and II cardiac pulsations appeared to be separated by a brief period of diastasis or cardiac rest. In this case, as shown in Fig. 9A, the sensors revealed a mixture of three consecutive heartbeat patterns, i.e. anterograde I, diastasis and anterograde II. This shows that the weak anterograde II type of cardiac pulsation can occur quite independently between the brief periods of diastasis. The results obtained using nanotechnological methods, provide evidence that the physiologically important switchover between the high pressure (anterograde I) and low pressure (anterograde II) heartbeats depends on the sudden opening of the conical heart chamber. A sudden "leakage" of haemolymph from the middle of the tubular dorsal vessel could account for the sudden fall of intracardiac pressure during an anterograde II pulsation. Accordingly, the physiological difference between anterograde I and II heartbeats is quite simple: the former pumps haemolymph through the aortan barrier of pressure into the head and thorax; the latter enhances the circulation of haemolymph within the abdominal cavity via the suddenly opened apertures in the conical chamber.

\section{The true retrograde heartbeat in Drosophila}

In most insects, the narrow thoracic aorta can occasionally support the anterograde beating of the heart, but not the backward directed beating. Thus, the retrograde heartbeats are associated with the abdominal circulation of haemolymph. The retrograde cardiac peristalsis is usually absent in mobile larval stages, where abdominal haemolymph is circulated by movements of the body, extracardiac pulsations or intestinal peristalsis. The rates of retrograde pulsations are half those of anterograde ones and often occur as individual systolic strokes (for references see discussion). In most insects, both antero- and retro-pulsations of the heart show peristaltic waves, moving sequentially from one metameric myocardial segment to the next. The present experiments on Drosophila, however, revealed different rules of cardiac performance. 


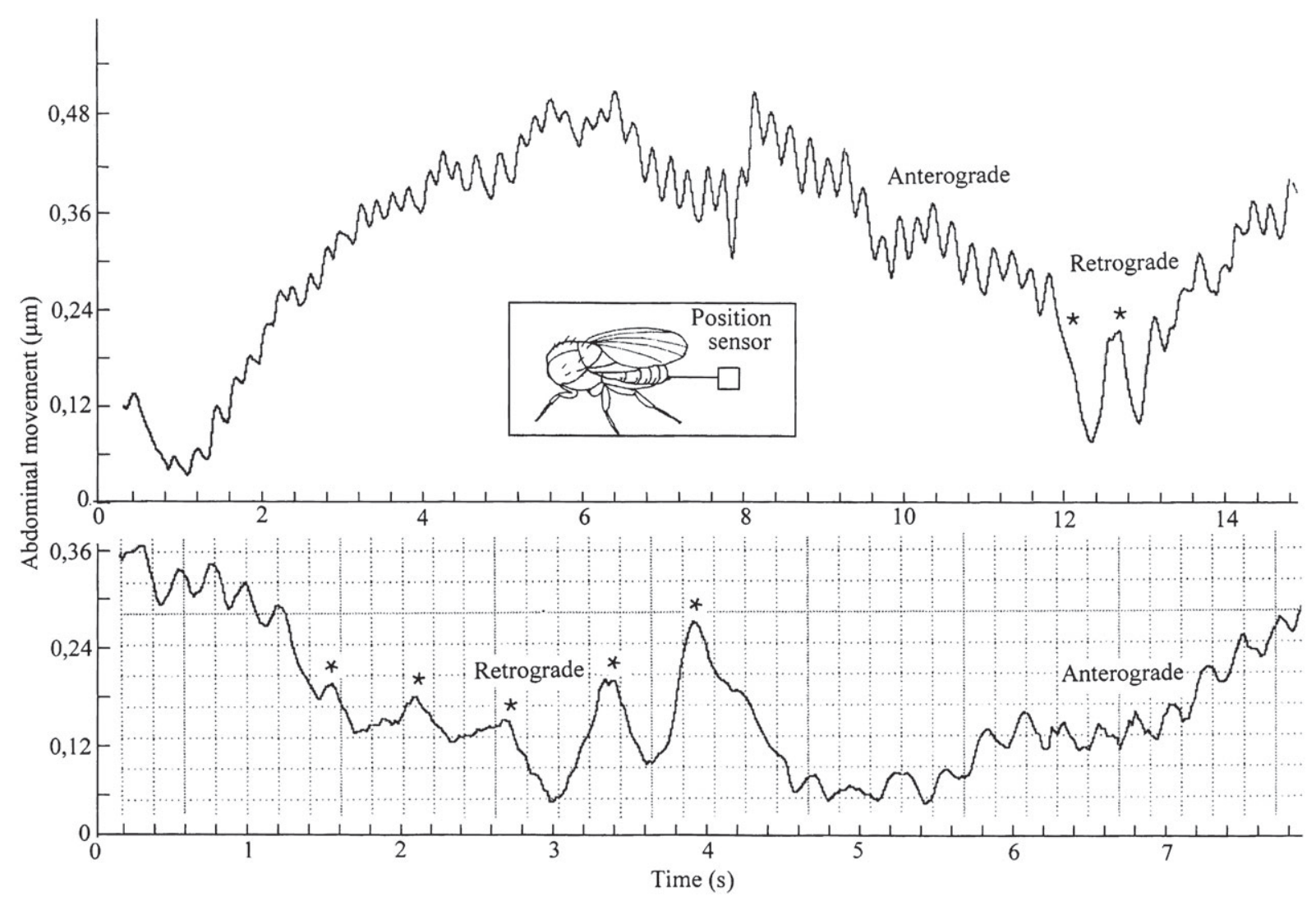

Fig. 10. Impact of heartbeat on the movement of the terminal abdominal segment at the time of low extracardiac interference, revealed by a high resolution posimetric recording (inset) of a young female of $D$. melanogaster, about $5 \mathrm{~h}$ after decapitation. Note that the fast anterograde pulsation caused a 30 to 60 nanometer decrease in the length of the abdomen, while the retrograde pulse (asterisks) caused a 120 nanometer increase in length.

The rates of the two anterograde heartbeats ( $4 \mathrm{~Hz}$ or up to $6.5 \mathrm{~Hz}$ under stress) may be too fast for the peristaltic segmental propagation of the systolic waves. As indicated above, anterograde I heartbeat directs haemolymph into the head and thorax, and anterograde II into the abdomen. Thus, if there is a normal retrograde heartbeat, as in other insects, what would be its physiological function?

This was investigated by a retrospective search for a slower retrograde heartbeat in all the cardiac records. It appears that: (a) A retrograde heartbeat is incompatible with bouts of strong anterograde I heartbeats; (b) Symptoms of retrograde heartbeat are due to the slow cardiac contractions ( 1 to $2 \mathrm{~Hz}$ ) of considerably larger amplitude occurring usually in the form of individual or twinned systolic contractions during the periods of diastasis; (c) These, tentatively retrograde cardiac pulses, were abolished during periods of anterograde II pulsation. In some cases there was an interplay of four different heartbeat patterns; anterograde I, anterograde II, diastasis and the retrograde heartbeat. Examples of this type of composite heartbeat pattern are presented in Fig. 9B. This thermographic record reliably displays the details of individual systolic strokes of the heart in adult Drosophila. In order to enhance the recording of the retrograde heartbeat the sensor was placed at the end of abdomen, over the terminal chamber of the heart. Results of previous experiments indicate that unlike the anterograde heartbeat, the retrograde heartbeat was always most pronounced at the end of the abdomen, which is also indicated by the traces in Fig. 9B.

The retrograde cardiac contractions are clearly distinguished from anterograde pulsations by their relatively large amplitude (almost 10-fold larger than anterograde) and approximately 2-fold smaller frequency (see Fig. 9B). Similar series of individual retrograde cardiac pulsations occur in the pupal or adult stages of most insects (see discussion). The switches between the reciprocal antero- and retro- directions are very rapid. This is documented in the final part of the trace in Fig. 9B by a quick interplay between anterograde II and retrograde systolic contractions (seconds 26 to 30). Using only the frequency, the true retrograde heartbeat $(1-2 \mathrm{~Hz})$ can be easily confused with extracardiac pulsations of the visceral organs or ventral diaphragm (1-2 Hz; see Fig. 2) and strong extracardiac pulsations in haemocoelic pressure $(0.5-2 \mathrm{~Hz}$; see Figs 4 and 5). Thus in the absence of a good knowledge of the effect of interference from extracardiac pulsations, the identification in vivo of the true retrograde cardiac pulsations in Drosophila is impossible.

Physiological mechanisms responsible for the switch between anterograde I (thoracic), anterograde II (abdominal) and retrograde (reverse abdominal) cardiac functions represent a new feature in insect cardiology. The switching period between anterograde I and II heartbeats is relatively long and of the order of 5 to $10 \mathrm{~s}$, which is very different from the millisecond excitation action 


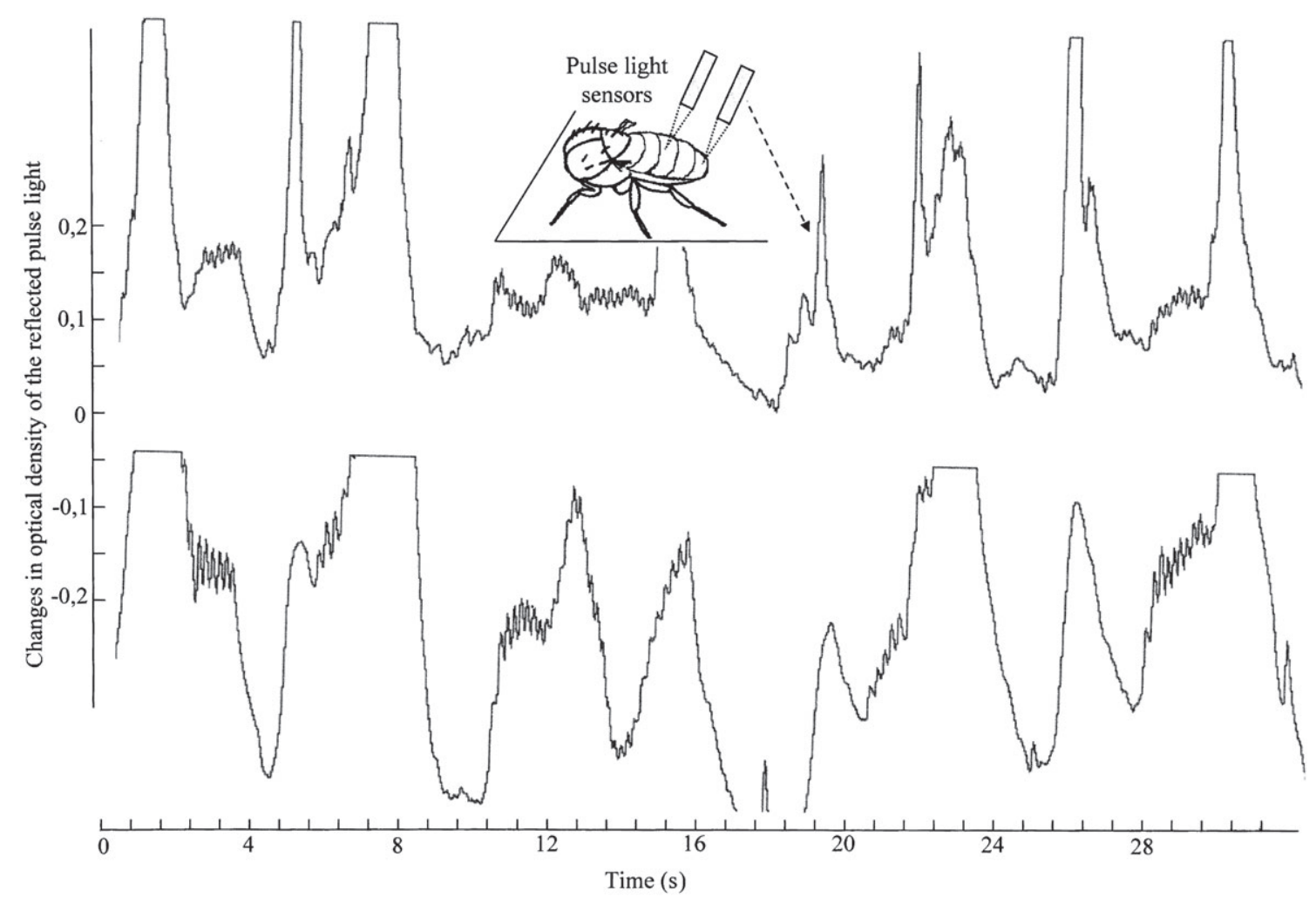

Fig. 11. Simultaneous recordings of two pulsed-light sensors, one focused on the pericardial region of the 3rd abdominal tergite and the second on the dorsal side of the tip of abdomen (just above the terminal heart chamber; see inset). The traces are of a young (3-day-old) female of D. melanogaster, 2-3 h after decapitation and recorded at $25^{\circ} \mathrm{C}$.

potentials generated by myogenic pacemakers. The increases and decreases in optical density indicate tonicor tetanic-like contractions and relaxations of the myocardium or muscular ligaments of the conical chamber. The available evidence indicates that during the anterograde I haemolymph pumping into the head, the conical chamber is invariably contracted (high optical density) and the apertures tightly closed. Conversely, during anterograde II abdominal circulation, the conical chamber is relaxed or expanded for 5 to $10 \mathrm{~s}$ (low optical density) and the apertures are widely open. This configuration is suitable for anterograde II cardiac pulsation, which causes haemolymph to circulate from the rear towards the base within the heart and backwards through the abdominal cavity. Alternatively, during the retrograde heartbeat, haemolymph is sucked into the heart lumen through open apertures. In this case haemolymph circulates in the opposite direction, i.e. from the base to the tip of the abdomen within the heart and forwards through the abdominal cavity. This accords with the complex physiological relationships that occur during heartbeat reversal in Drosophila. However, the physiological nature of the switching mechanism that causes 5 to $10 \mathrm{~s}$ contractions and relaxations (closure and opening of the apertures) of the conical chamber remains to be investigated.

\section{Posimetric determination of intracardiac haemolymph flow}

Although a single thermographic or optical sensor cannot reliably determine the direction of haemolymph flow within the heart, the reciprocal directions of the heartbeat flow can be indirectly determined by the forward or backward movements of the flexible distal abdominal segment. In principle, retrograde and anterograde cardiac pumping of haemolymph should cause respectively a prolongation and retraction of the distal abdominal segment. Measurements of these tiny movements required the use of very sensitive, semiconductor strain-gauges, capable of detecting movements with nanometer accuracy. The posimetric sensors were attached to the end of the abdomen of a decapitated adult Drosophila by very thin metallic wires $(50 \mu \mathrm{m} \mathrm{O}$. D., see inset in Fig. 10). Flexible membrane construction of these sensors enabled simultaneous recording of large extracardiac pulsations as well as million-fold smaller movements associated with the heartbeat.

Fig. 10 (upper trace) documents the above by a detailed trace of the movements of the end of the abdomen over a period of a $15 \mathrm{~s}$ heartbeat episode, part of a prolonged (3 h) posimetric recording. The data show intimate details of a continuous anterograde heartbeat, starting at a frequency of $5 \mathrm{~Hz}$ and terminating at a somewhat lower rate of $4 \mathrm{~Hz}$. The slowly decreasing rate of anterograde heartbeat is a common physiological feature of most insect species. It is not possible to determine the exact delimitation of the boundary between anterograde I and II, because the distal end of the abdomen is the place where these pulsations originate.

The most important features in Fig. 10 (upper trace) are the two large systolic peaks (indicated by asterisks) 


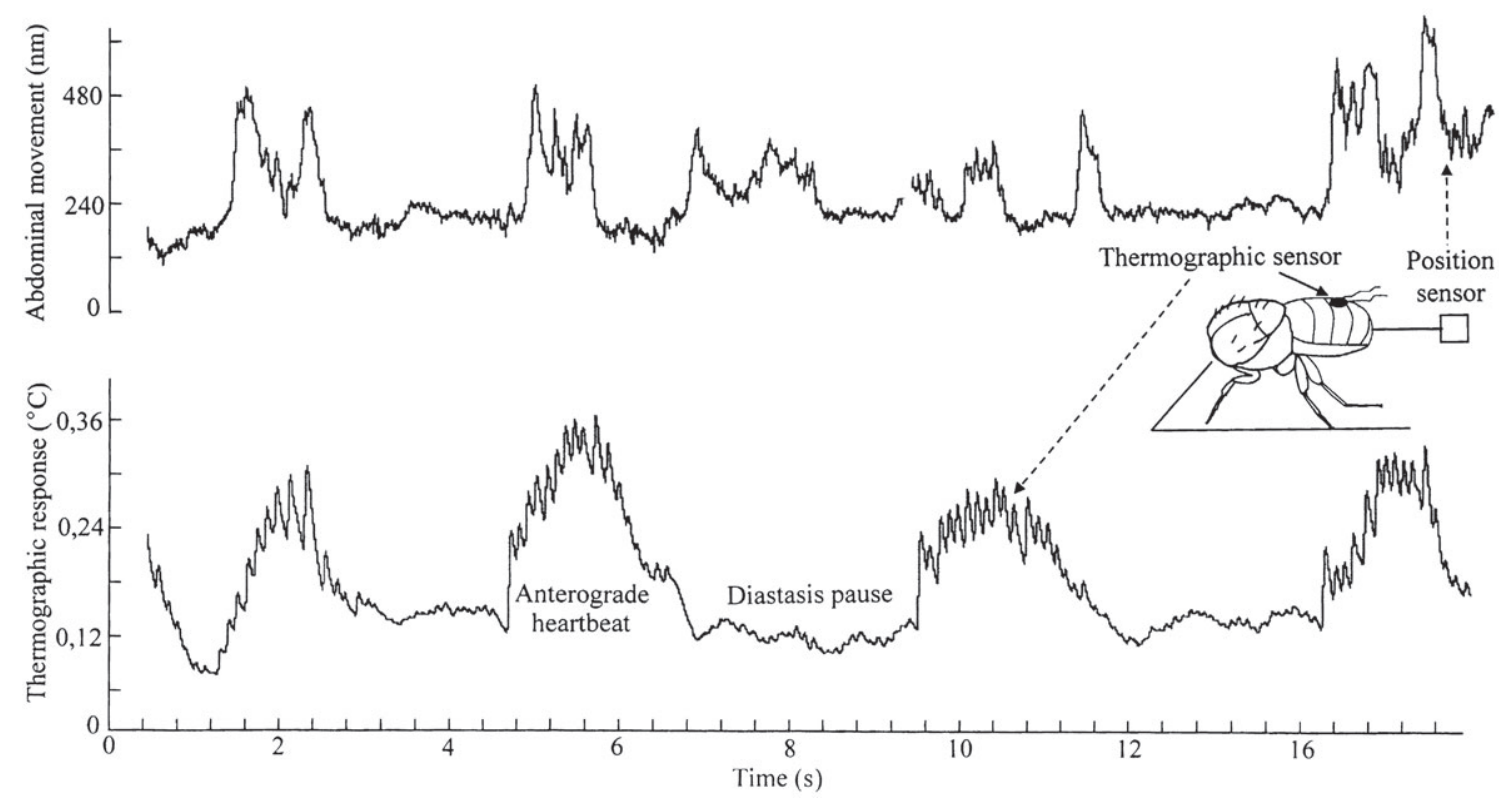

Fig. 12. Simultaneous recordings by a position sensor attached to the tip of the abdomen and a thermographic sensor to the 3rd abdominal tergite (inset) of a young female of D. melanogaster, $3 \mathrm{~h}$ after decapitation, at $26^{\circ} \mathrm{C}$.

recorded between 12 and 13 s. They are clearly distinguished from previous anterograde pulses by longer duration and larger amplitude. In marked contrast to the $4 \mathrm{~Hz}$ anterograde pulses, which are associated with 30-60 nm shrinkages of the abdomen, the two large systolic pulses, marked by asterisks, are associated with a $120 \mathrm{~nm}$ prolongation of the abdomen. Therefore, it is likely that the two reciprocal peaks record the effects of the retrograde, backward oriented haemolymph stream expelled from the orifice of terminal ampulla of the heart. This is clear evidence for the hitherto unknown, true retrogade cardiac pulsation of the adult heart in Drosophila. This conclusion can be further corroborated by the high resolution, lower trace in Fig. 10, which shows perfectly amplitude and frequency in a series of 5 retrograde cardiac pulses (asterisks) intercallated between the faster, positively anterograde ones.

After posimetric identification of the rates, sizes and shapes of the real retrograde pulsations ( $n=12$ records, duration 2-3 h), retrospective search was initiated for similar cardiological features in other thermographic and optoelectronic records. It appears that the positively identified retrograde heartbeat occurred only rarely or not at all. In general, there are no records of regular alternations between anterograde and retrograde heartbeats unlike in other insect species. When present, the true retrograde pulsations emerged from the diastasis periods, especially in young decapitated gravid females $(n=11$ records; duration 2-5 h). In most cases the retrograde heartbeat appeared only in the form of large, individual or twinned systolic peaks (see Fig. 10 for illustration; $n=8$ records; duration 3-5 h). Longer consolidated series of retrograde pulsations, such as the one shown in Fig. 9B, were relatively rare. Decapitated adult males or young unfed females ( $n=18$ records; duration $2-4$ h) with mainly anterograde II heartbeat, did not exhibit true retrograde pulsations. In this case, retrograde circulatory functions in the abdomen were carried out by the more efficient, anterograde II heartbeat.

\section{Simultaneous recording of heartbeat by two sensors}

In Drosophila, the relatively small size of the abdomen, combined with the synchronic heartbeats, complicated the cardiographic measurements. The use of touch-free, optocardiographic sensors, which have proved to be very good in studies on other insects, did not give satisfactory results. Fig. 11 shows that there was usually an ocean of intervening extracardiac abdominal movements, among which the heartbeat $(3.5 \mathrm{~Hz}$, small amplitude) is only apparent for short intervals. It is noteworthy that the large extracardiac peaks in Fig. 11 are systemic, not peristaltic. This means that the changes arrive at both sensors at the same moment. A high resolution analysis of this and similar optical records failed to show peristaltic propagation of the systolic waves of the heart. There were almost immediate, synchronized systolic contractions at the end and in the middle of the heart (less than $25 \mathrm{~ms}$ difference). This failure was not due to artifacts produced by interference between two or more light sensors situated close to each other (which can respond almost as a single sensor). The advanced pulsed-light technique uses electronically filtered light beams of different frequencies for each radiator and receiver. Failure to determine peristaltic heartbeat by optoelectronic methods (Fig. 11) prompted the study of the nature of $4 \mathrm{~Hz}$ anterograde heartbeat in Drosophila using other recording techniques.

\section{Combined use of thermographic and position sensors.}

The results of the simultaneous use of thermographic and posimetric methods can be exemplified by the records presented in Fig. 12. They show close correlations between the directions of cardiac haemolymph flow and the contractions or prolongations of the terminal 


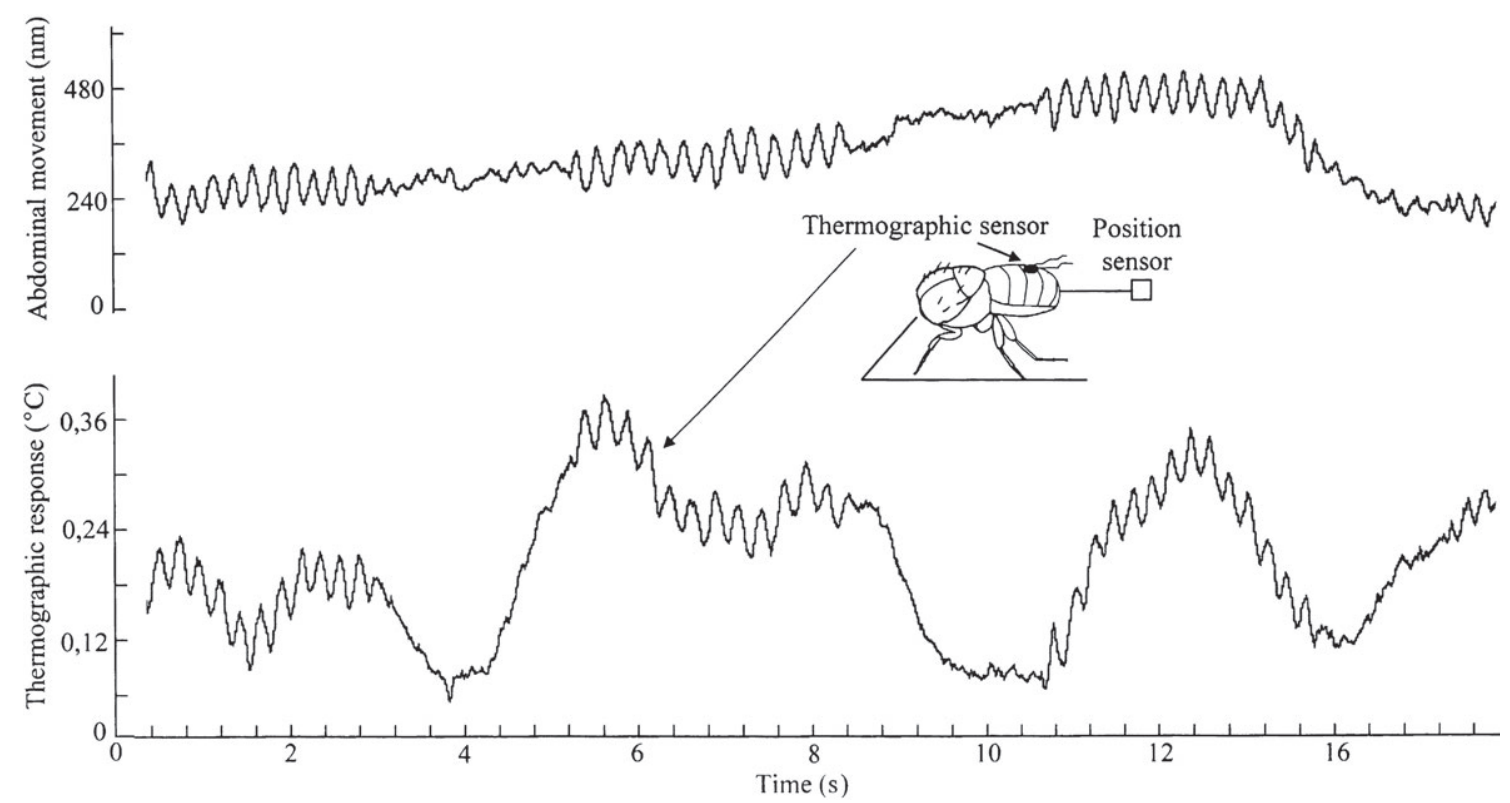

Fig. 13. High resolution, simultaneous recordings of the anterograde heartbeat during periods without extracardiac interference, using posimetric and thermographic sensors (inset) attached to an adult male of D. melanogaster ( $2 \mathrm{~h}$ after decapitation, $\left.25^{\circ} \mathrm{C}\right)$.

abdominal segment (inset). The lower, thermographic record in Fig. 12 shows 4 common episodes of anterograde I heartbeat separated by 5 to $8 \mathrm{~s}$ periods of cardiac rest. The upper, posimetric record shows that each bout of anterograde I heartbeat is associated with a $240 \mathrm{~nm}$ abdominal retraction. Obviously, this was due to pumping haemolymph from the tip of abdomen into the head and thorax. Other similar records $(n=12)$ revealed that the anterograde II cardiac pulsation produced a much smaller effect (not shown in Fig. 12). This may be explained by the fact that haemolymph in this case only circulated within the abdominal cavity. High resolution recordings of the combined posimetric-thermographic methods (Fig. 13) revealed that the effect of individual systolic contractions was rather small, resulting in a movement of the abdomen of only $100 \mathrm{~nm}$ or less.

The good resolution of individual systolic strokes in Fig. 13, made it possible to measure the velocity of propagation of the synchronic waves of anterograde cardiac contractions. It was anticipated that the velocity of synchronic propagation of one systolic-diastolic episode over all 4 abdominal segments would be faster than that of 4 separate peristaltic episodes travelling the same distance. The velocity of systolic contractions was determined between the posterior end (terminal chamber) and the proximal end (conical chamber) of the heart. The results of these studies are presented in Fig. 14 and were achieved by placing the smallest available thermographic sensor just above the conical chamber (see inset). Irrespective of the actual principle of heartbeat propagation, the measurements revealed consistently that the systolic anterograde contractions originated in the terminal chamber of the heart, probably in a terminal regulatory nodus analogous to that of other species (see Discussion).

Theoretically, the myogenic action potentials of the posterior regulatory nodus should excite anteriorly moving waves of systolic contractions in the myocardium cells of all segments of the heart, similarly like the waves of systolic contractions starting at the tip of the human heart. It is unknown whether this systolic contraction of insect heart continues along the aorta towards the head. It is more probable, however, that the contraction stops at the conical chamber. The records in Fig. 14 show a distinct but slight retardation in the systolic contractions on their way from the terminal to the proximal chamber of the heart in Drosophila. The actual time decrement is close to $95 \mathrm{~ms}$. When this is calculated per unit of time, the distance of $0.9 \mathrm{~mm}$ traversed within $95 \mathrm{~ms}$ indicates an approximate velocity of the systolic contractions, at $23^{\circ} \mathrm{C}$, of $9.5 \mathrm{~mm}$ per second. Surprisingly, a velocity of $9.5 \mathrm{~mm}$ per second for the anterograde synchronic heartbeat in Drosophila is not exceptional. It falls within the common range of velocities recorded for the propagation of peristaltic cardiac waves in most other insects.

\section{Sensor techniques combined with visual observations}

As decapitated adults of Drosophila remain motionless it is possible to make in vivo observations of the heart under a stereomicroscope. The rate of the heartbeat is relatively fast and difficult to measure. In addition, it is not possible to see the direction of movement of haemolymph within the lumen of the heart. This makes optical determination of the forward or backward oriented directions of haemolymph flow within the heart lumen almost impossible. Under certain circumstances, however, visual observations of cardiac movements can help to verify or disprove interpretations reached using the sensors.

The common heartbeat of most insects is based on the peristaltic principle that each myocardium segment can perform both systolic contractions and diastolic dilations. The results presented here indicate that the segmentally propagated peristaltic waves recorded in Drosophila represent only the relatively slow retrograde heartbeat. On 


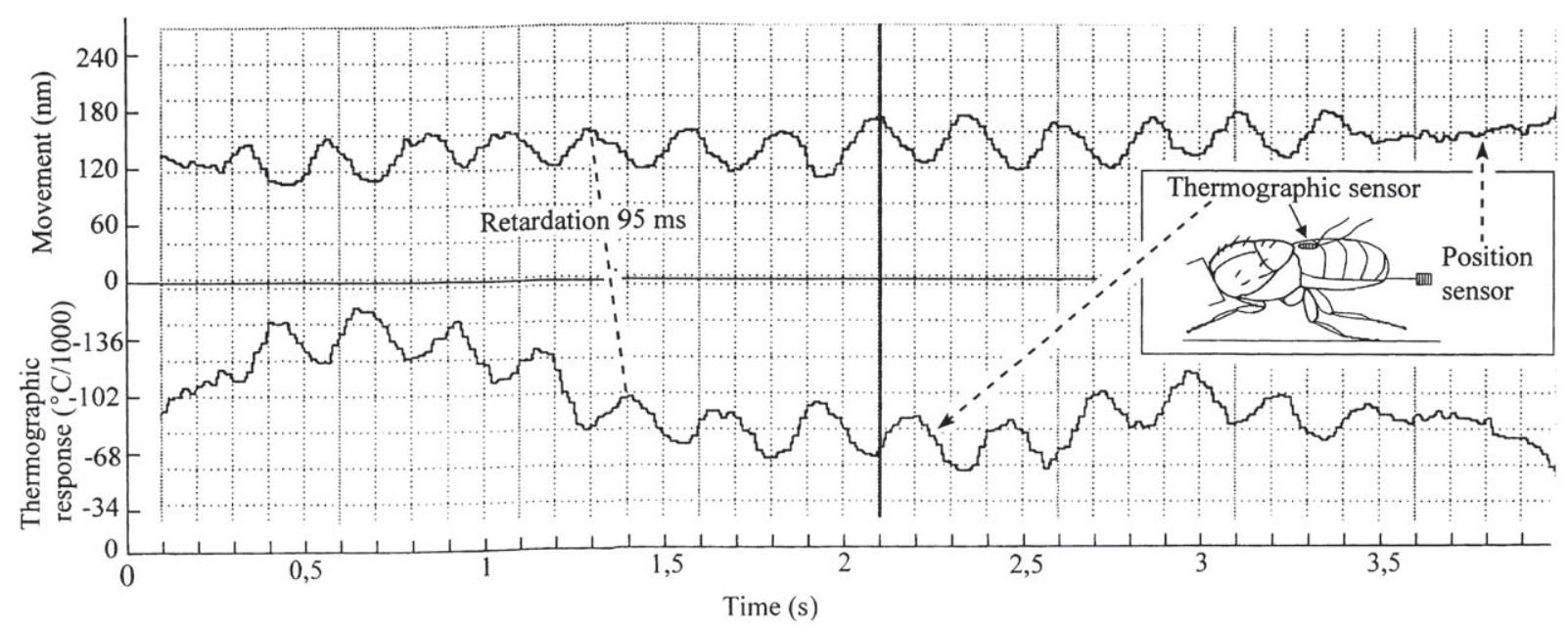

Fig. 14. High resolution, combined posimetric and thermographic records (inset), illustrating the retardation of the anterograde systolic contractions from the end to the base of the abdomen (7-day-old female D. melanogaster, $3 \mathrm{~h} \mathrm{after} \mathrm{decapitation} \mathrm{at} 23^{\circ} \mathrm{C}$ ).

the other hand, the much faster and far more efficient anterograde I and II heartbeats are not peristaltic. Under a microscope, one can see that synchronous contractions occur along the whole length of the heart, without segmentally arranged systolic waves. In order to clearly distinquish this from the common peristaltic principle, we propose to use the term synchronic principle or synchronic cardiac mechanism. According to the data in Fig. 14 , the synchronic contractions spread from the terminal to conical heart chamber in $95 \mathrm{~ms}$ and thus the maximum physiologically permissible rate of anterograde heartbeat is likely to be close to 10 strokes per second $(10 \mathrm{~Hz})$ at room temperature.

Visual observations and video recordings can be enhanced by injecting black india ink into the haemocoele, which is ingested by and stains the pericardial cells black and so reveals the movements of the heart, as illustrated in Fig. 1. Visual observations of the fast anterograde heartbeat revealed that the entire heart moved in synchrony, without individual peristaltic contractions, confirming the synchronic nature of the heartbeat. In this case, the shape of the heart is conical with the narrow end at the rear (Fig. 15). This arrangement certainly facilitates the pumping of haemolymph forwards into the elastic aortan tube. It is concluded, therefore, that the synchronic contractions increase also the amplitude of the oscillations in intracardiac pressure, which is essential for the functioning of the ostial valves.

There are 4 pairs of ostial valves in adult Drosophila, functioning as passive, mechanically operated, incurrent valves. The mechanism is based on similar hydraulic principle operating the valves in the human heart. During the systolic pressure peak the valves are closed by the increase in intracardiac pressure. Alternatively, during the diastolic decline in intracardiac pressure, the ostial valves open for a few milliseconds and haemolymph is sucked into the heart. According to our estimates, the pumping efficiency of each synchronic stroke of the heart could be 5 -fold greater than a peristaltic wave composed of 5 metameric episodes. The great selective advantage of the newly described synchronic heartbeat mechanism in Drosophila, whose function is analogous to the contractions that start at the tip of the human heart, may depend on overcoming the increased pressure associated with a narrow elastic aorta (analogous to that associated with the arteries and capillaries in the human vascular system).

Visual observations also threw some light on the problem related to the periodical switch (5 to $10 \mathrm{~s}$ intervals) between high and low optical densities of the light passing through the conical chamber. These contractions and dilatations of the conical chamber are crucial for understanding heartbeat reversal in Drosophila. The data obtained from the sensors and visual observations, enabled us to reach the final conclusions (see Fig. 15): 1. The conical chamber contracts and the apertures constrict during the 5 to $10 \mathrm{~s}$ bouts of vigorous anterograde I pulsations, when haemolymph is pumped forwards into the aorta, under increased intracardiac pressure, and; 2 . The conical chamber expands and the apertures open widely during the anterograde II pulsation, when haemolymph leaks out of the heart and circulates backwards through the haemocoelic cavity. The periodically alternating contractions (higher optical density, closed apertures) and expansions (lower optical density, open apertures) of the conical chamber undoubtedly represent the most important cardiac phenomenon in Drosophila and possibly other insects.

\section{Physiological considerations}

The results of this study are summarised in Fig. 15. There are 3 main categories of cardiac functions in adult Drosophila: 1. Anterograde I heartbeat, which circulates haemolymph mainly in the thorax and head and is manifested by vigorously pushing abdominal haemolymph forwards into the aorta under increased intracardiac pressure $(\approx 4 \mathrm{~Hz}$; ligaments of conical chamber contracted, apertures closed, mechanism synchronic); 2. Anterograde II heartbeat, which circulates haemolymph only in the abdomen and is manifested by the pumping of haemolymph from the rear to the base of abdomen $(\approx 3.5 \mathrm{~Hz}$; ligaments of conical chamber expanded; apertures open, 


\begin{tabular}{|c|c|c|c|c|c|}
\hline Heartbeat & Mechanism & Frequency & Circulation & onical chamb & Apertures \\
\hline Anterograde I & Synchronic & $\sim 4 \mathrm{~Hz}$ & Thoracic & Contracted & Closed \\
\hline Anterograde II & Synchronic & $\sim 4 \mathrm{~Hz}$ & Abdominal & Dilated & Open \\
\hline Retrograde & Peristaltic & $1-2 \mathrm{~Hz}$ & Abdominal & Dilated & Open \\
\hline
\end{tabular}
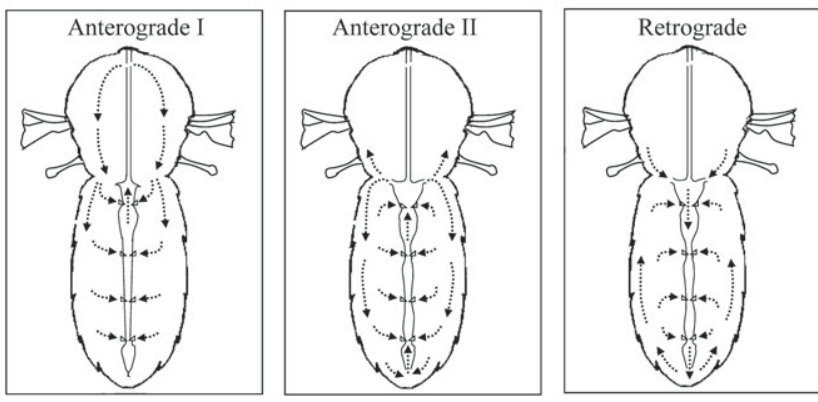

Fig. 15. Schematic outline of circulatory pathways during periods of anterograde I, anterograde II and retrograde heartbeat in adult D. melanogaster.

mechanism synchronic), and; 3. Retrograde heartbeat, which circulates haemolymph only in the abdomen and is manifested by the pumping of haemolymph reciprocally from the base towards the end of the abdomen $(\approx 1-2 \mathrm{~Hz}$ or individual; ligaments of conical chamber expanded, apertures open, mechanism peristaltic). Superficially, this 3-phasic cardiac schedule recorded in Drosophila differs from the common, 2-phasic heartbeat in other insects in that there is a second adominal, anterograde II pulsation, which results in a very efficient, synchronic cardiac mechanism.

Further physiological implications of the results obtained in this study can be briefly described as follows: (a) Enhancement of the circulatory functions in Drosophila has been achieved by a more efficient, synchronic anterograde I heartbeat; (b) Haemolymph that is pushed towards the head during anterograde I pulsation, can leave the narrow aortan tube through excurrent thoracic ostia and circulate backwards through thoracic venous channels; (c) Haemolymph currents within the aorta seem to be unidirectional, exclusively anterograde, enhanced eventually by autonomic pulsation of the distal, ventrally directed, abdominal part of the aorta; (d) Haemolymph coming back through the thoracic venous channels mixes with the stream of haemolymph produced by the anterograde II pulsation, which drives it back into abdominal circulation; (e) The main function of the backward oriented peristaltic retrograde heartbeat is that it removes haemolymph that has accumulated in venous channels by sucking it into the heart through the open apertures in the conical chamber.

Thus, the physiological novelty of the described circulatory pathways in Drosophila are the actively controlled switches between very efficient thoracic (anterograde I) and abdominal (anterograde II) cardiac pulsations. This is associated with the development of a synchronic heartbeat mechanism and hitherto unknown, tonic or tetanic contractions (closed apertures) and relaxations (open apertures) of the conical heart chamber. The physiological mechanism regulating the contractions of the conical chamber is still unknown and remains to be investigated. I am convinced that it is associated with the large muscular attachments that stretch from the proximal part of the conical chamber laterally and anchor it to the body wall between the thorax and abdomen. The fact that an invertebrate animal, such as Drosophila, evolved a compact heart (conical chamber) with atrium and synchronic cardiac function analogous to that of the human heart, may open new avenues for future research in comparative animal cardiology.

\section{DISCUSSION}

Physiological functions of the heart in the living body are intimately connected with metabolism. The most important metabolic factors affecting the heartbeat are feed-back responses mediated via haemolymph from: (a) respiratory metabolism (carbonate ions); (b) intermediary metabolism (norepinephrine, serotonin); (c) muscle activity (pyruvate, lactate); (d) digestion (glutamate); (e) excretion (osmotic pressure, salts) and other factors like ionic and water balance. It is well known that similar factors affect the rhythmicity of the human heart (Sláma, 2005; Sláma et al., 2006). There are also similarities in the embryonic formation of the heart, common progenitor cells, similar genes (tinman) and other characteristics of Drosophila and human hearts (Bodmer, 1995; Bodmer \& Venkatesh, 1998; Bodmer et al., 2005; Akasaka et al., 2006). Ten years ago, when Bodmer \& Venkatesh (1998) described the above similarities, they were puzzled by the similarity of these two functionally divergent systems. The present finding of a compact conical chamber and synchronic heart in Drosophila, with the pacemaker regulatory nodus in the terminal chamber of the heart, substantiates their conclusions about the genetical and structural analogies between the heart of Drosophila and man (Bodmer \& Venkatesh, 1998) and, what is more important in terms of this study, the findings may extend their genetical arguments to functional similarities.

In terms of comparative physiology, human and insect hearts can be regarded as involuntary, myogenic systems whose rhythmicity is generated within a special atrioventricular nodus in humans or within a posterior regulatory nodus in the heart ampoule in insects (Sláma, 2006). Neuropeptides do not have any direct effects on the human heart like the various reports describing "potent" cardiostimulating effects of neuropeptides on the insect heart (for references see Sláma, 2005). My recent study on the heart of a lepidopteran insect, Manduca sexta, confirm the existence of cardiological similarities between vertebrates and invertebrate animals (Sláma, 2006). For example, the removal of all essential nervous centers, including the brain or terminal abdominal ganglionic mass, had no effect on the pattern of heartbeat reversal. In addition, both anterograde as well as the opposite, retrograde peristaltic waves were both initiated by the posterior regulatory nodus in the terminal heart ampoule, which is equivalent (Sláma, 2006) to the atrio-ventricular nodus of the human heart (Bodmer et al., 2005). Preliminary experiments involving sectioning the heart of Droso- 
phila revealed that the pacemaker rhythmicity is also regulated from the terminal chamber (Sláma, 2009, unpubl.).

Many years ago, it was assumed that the regulation of insect hearts was completely different from that in vertebrates (Ai \& Kuwasawa, 1995; Dulcis et al., 2001). These authors believed that the reversal of the insect heartbeat was controlled by two neurogenic regulatory pacemakers, one in the front for the retrograde and the other in the tail for the anterograde direction (for a review of a similar topic see Johnson, 1977). There are reports suggesting the existence of a special cardiac pacemaker in the frontal part of the nervous system in Bombyx (Ai \& Kuwasawa, 1995) or neurogenic pacemakers in the posterior, terminal abdominal ganglionic mass in Manduca (Dulcis et al., 2001). Recent studies involving sectioning of the heart and removal of essential nervous ganglia revealed that the indigenous rhythmicity and periodic heartbeat reversal were absolutely independent of the presence or absence of these essential nervous centers (Sláma, 2006).

Unlike in human pharmacology and cardiology, some authors report inducing potent cardiostimulating effects by applying neuropeptides to insect hearts incubated in vitro (Tublitz \& Truman, 1985; Tublitz, 1989; Veenstra, 1989; Nichols, 1999; Zornik, 1999; for review see Miller, 1985, 1997). A reinvestigation of the effects of proctolin, crustacean cardioactive peptide (CCAP) and corazonin indicated that these peptides have no effect on the rate of heartbeat in living insects (Sláma, 2004; Sláma \& Rosiński, 2004). In addition, the neuropeptides that are "cardioactive" in vitro assays, using the heart of the American cockroach (Tublitz \& Truman, 1985; Veenstra, 1989), do not affect the heartbeat of a living American cockroach (Sláma et al., 2006). These results are consistent with the assumption of Bodmer et al. (2005) that insect and human hearts should respond similarly to regulatory substances.

At this point let us again consider the heart of Drosophila. The results of in vivo recordings using several electrocardiographic techniques reveal a number of physiologically important facts. The newly discovered system of compact heart chamber and synchronic, instead of peristaltic regulation of the heartbeat, apparently represents an evolutionary adaptation to increased circulatory demands. This may be connected with the extremely high circulatory and respiratory demands of Diptera and other endopterygote insects, which fly. The most essential physiological features of the synchronic cardiac function are: (a) Excitation action potentials, originating by depolarization potentials in the myocardium cells of the posterior regulatory nodus, excite systolic contractions that spread along the entire length of the heart, without segmental diastolic relaxations; (b) The period required for the unified, synchronic systolic/diastolic pulsation to traverse the heart is estimated to be 4- to 5-fold shorter than that of 4 to 5 successive peristaltic episodes; (c) The relatively fast rates of anterograde synchronic heartbeat $(4 \mathrm{~Hz}$ or more), enable the synchronic mechanism to induce a new contraction before the last one has reached the aorta (maximum $10 \mathrm{~Hz}$ ); (d) Combined contractions of several myocardium segments is substantially more efficient in pushing haemolymph into the narrow aorta than the peristaltic system; (e) Hearts specifically adapted to unidirectional, anterograde performance are most probably unable to run synchronically also in the opposite direction; (f) The last statement is corroborated by the presence of one-way valves at the orifice of the aorta in many insect species (see Jones, 1977; Tögel \& Pass, 2006).

The synchronic, anterograde I heartbeat is specifically used for enhancing haemolymph circulation to the head and thorax. The synchronic, anterograde II heartbeat effectively supplements the less efficient and relatively slow peristaltic retrograde heartbeat. Thus the 4-phasic pattern of heartbeat reversal in Drosophila deviates from the common tri-phasic cardiac pathway of other insects (anterograde, diastasis, retrograde) in its synchronic mechanism and the newly established, anterograde II circulatory pathway (see Fig. 15).

The purely abdominal anterograde heartbeat is a new phenomenon in insect physiology and is based on a hitherto unknown, actively operated opening and closing of apertures in the conical chamber. The mechanism that actively regulates opening and closing of the apertures is significantly different from the passive, mechanical operation of the one-way ostial valves. The latter are simple membrane valves that close when the pressure increases during systole and open when the pressure falls during diastole (Wigglesworth, 1965; Jones, 1977; Tögel $\&$ Pass, 2006). The apertures in the conical chamber of Drosophila heart, however, remain open or closed irrespective of the number of systolic/diastolic waves passing through.

The ostial valves in insect hearts are distinguished by their embryonic origin, location and specific anatomical structure (Tögel \& Pass, 2006). In adult Drosophila, the origin and nature of the 4 pairs of ostial valves are well documented in the literature (Rizki, 1978; Curtis et al., 1999; Bodmer et al., 2005; Zeitouni et al., 2007). Recently, Wasserthal (2007) reported finding of another pair of ostial apertures in the anterior part of the conical chamber. I am convinced that Wasserthal (2007) actually found another type of aperture. The evidence provided by the electronic sensors indicate that these apertures represent a new type of actively operated, slit-like diaphragms within the wall of the heart, which are closed or opened for several seconds by tetanic contractions or relaxations of the conical chamber, or its proximo-lateral muscular attachments, respectively.

The existence of actively regulated switching between closed and open apertures in the conical chamber, i.e. between thoracic and abdominal circulation, may open new avenues in cardiological insect research. Physiological mechanisms regulating these switches in circulation remain to be elucidated. It is possible that they are regulated by metabolic feed-back factors and neurogenic factors related to innervation of the conical chamber (Dulcis \& Levine, 2005). Unfortunately, the recordings are of immobile, decapitated flies. It is likely, however, that a flying or running fruitfly would use the most effi- 
cient and appropriate functions of the synchronic circulatory systems.

A synchronic heartbeat need not be limited to Drosophila. We have recently investigated the heartbeat of the American cockroach, the species used for most investigations on the circulatory system of insects (see Krijgsman, 1952; Wigglesworth, 1965; Johnson, 1977; Miller, 1979, 1985). The in vivo optocardiographic recordings (Sláma et al., 2006) of the heart of an intact American cockroach indicate relatively fast heartbeat rates (close to $1 \mathrm{~Hz}$ ), plus the characteristic features of the unidirectional, synchronic cardiac mechanism.

In the absence of posimetric and thermographic recordings (see Figs 10, 12, 13, 14), it is only possible to speculate about the forward or backward directions of the intracardiac haemolymph currents. A previous optoelectronic study of immobile pharate adults of Drosophila, still enclosed within the puparium (Sláma \& Farkaš, 2005), revealed a relatively fast rate of anterograde heartbeat. Preliminary measurements on recently emerged flies usually indicate confusing, unidirectional anterograde heartbeat over prolonged periods of time. Other authors, also using optical methods and Drosophila, apparently experienced the same difficulties. Dulcis \& Levine (2005), for example, also identified the faster heartbeat rates as anterograde and the slower rates as retrograde. The conclusions of Sláma \& Farkaš (2005) and Dulcis \& Levine (2005) are consistent with all the heartbeat data previously obtained in studies of a number of other insect species (reviews by Wigglesworth, 1965; Jones, 1977; Miller, 1997).

Following the unequivocal identification of anterograde I $(\approx 4 \mathrm{~Hz}$, higher transmittance) and anterograde II $(\approx 3.5$ $\mathrm{Hz}$, lower transmittance) heartbeats, a large retrospective data analysis revealed that the heartbeats previously believed to be retrograde were in reality anterograde II heartbeats. The common alternation of the two anterograde heartbeats at 5- to 10 -s intervals accords well with previous observations by other authors, who record heartbeat reversal (Dulcis \& Levine, 2005; Wasserthal, 2007). The present evidence based on the use of various electrocardiographic sensors demonstrates, however, that true peristaltic retrograde heartbeat occurs rather sporadically, mostly in the form of individual or twin peaks (Figs 7, 9, 10). These heartbeats were missed in previous studies due to confusion with the anterograde II heartbeat and because they are hidden among extracardiac peaks of similar frequency.

Recently, an extensive, in vivo study of heartbeat in adult flies of Drosophila, using transmitted IR radiation in combination with linear optosensor chip, was published by Wasserthal (2007). He reports conspicuous, 5 to $10 \mathrm{~s}$ switches between high and low optical densities of the tranmitted light. These switches were ascribed to heartbeat reversal. According to the results presented here the high (contracted conical chamber) and low optical transmittance (expanded conical chamber) have nothing to do with bi-directional heartbeat reversal, as they are both anterograde. This controversial interpretation may be attributed to Wasserthal (2007) not expecting that the apertures in the conical chamber could open and close. In contrast to all previously published cardiological data (review by Jones, 1977; Miller, 1997), it was assumed that the rate of the forward oriented, anterograde heartbeat was slower than that of the retrograde heartbeat. This conclusion was supported by the argument that the slower heartbeat is more effective at pushing haemolymph into the head. Our results show that increase in circulation is always associated with an increase, not a decrease, in heartbeat in insects (cf. also Wigglesworth, 1965; Jones, 1977; Miller, 1997; Sláma, 2005, 2006). It is possible that the incorrect assignment to the direction of the heartbeat by Wasserthal (2007) might be associated with artifacts caused by recording the regressive, kick-back movements of the elastic wall of the heart.

ACKNOWLEDGEMENTS. We acknowledge with thanks the following colleagues: J. Fleischmanová for helpful discussions, M. Žurovec for help with the interpretation of the video records, and M. Šerý for supplying the photograph in Fig. 1. Corrections of English text by S. Phillips from Kensington, CA, USA are also highly appreciated.

\section{REFERENCES}

Ai H. \& Kuwasawa K. 1995: Neural pathways for cardiac reflexes triggered by external mechanical stimuli in larvae of Bombyx mori. J. Insect Physiol. 41: 1119 -1131.

Akasaka T., Klinedienst S., Occor K., Bustamante E.L., Kim S.K. \& Bodmer R. 2006: The ATP-sensitive potassium (KATP) channel-encoded dSUR gene is required for Drosophila heart function and is regulated by tinman. PNAS 103: 11999-12004.

BoDMER R. 1995: Heart development in Drosophila and its relationship to vertebrate systems. Trends Cardiovasc. Med. 5: 21-27.

Bodmer R. \& Venkatesh T.V. 1998: Heart development in Drosophila and vertebrates: conservation of molecular mechanisms. Devel. Genet. 22: 181-186.

Bodmer R., Wessells R.J., Johnson E.C. \& Dowse H.B. 2005: Heart development and function. In Gilbert L.I., Iatrou K. \& Gill S. (eds): Comprehensive Molecular Insect Science. Vol 2. Elsevier, Oxford, pp. 199-250.

Coats J.R. 1982: Insecticide Mode of Action. Academic Press, New York, London, 470 pp.

Curtis N.J., Ringo J.M. \& Dowse H.B. 1999: Morphology of the pupal heart, adult heart and associated tissues in the fruit fly, Drosophila melanogaster. J. Morphol. 240: 225-235.

Dulcis D. \& Levine R.B. 2005: Glutamatergic innervation of the heart initiates retrograde contractions in adult Drosophila melanogaster. J. Neurosci. 25: 271-280.

Dulcis D., Davis N.T. \& Hildebrand J.G. 2001: Neuronal control of heart reversal in the hawkmoth Manduca sexta. $J$. Comp. Physiol. (A) 187: 837-849.

Jones J.C. 1977: The Circulatory System of Insects. Charles C. Thomas, Springfield, $33 \mathrm{pp}$.

Klowden M.J. 2007: Physiological Systems in Insects. 2nd ed. Elsevier, Oxford, $688 \mathrm{pp}$.

KriJgsman B.J. 1952: Contractile and pacemaker mechanisms of the heart of arthropods. Biol. Rev. 27: 320-346.

MilLER T.A. 1979: Nervous versus neurohormonal control of insect heartbeat. Am. Zool. 9: 77-86. 
MiLleR T.A. 1985: Structure and physiology of the circulatory system. Comp. Insect Physiol. Biochem. Pharmacol. 3 289-353.

Miller T.A. 1997: Control of circulation in insects. Gen. Pharmacol. 29: 23-38.

Nation J.L. 2002: Insect Physiology and Biochemistry. CRC Press, Boca Raton, 485 pp.

Nichols R., Kaminski S., Walling E. \& Zornik E. 1999: Regulating the activity of a cardioacceleratory peptide. Peptides 20: $1153-1158$.

Ocorr K., Perrin L., Lim H.-Y., Qian L., Wu X. \& Bodmer R. 2007: Genetic control of heart function and aging in Drosophila. Trends Cardiovasc. Med. 17: 177-182.

Richter K. 1973: Struktur und Funktion der Herzen wirbelloser Tiere. Zool. Jb. Physiol. 77: 477-668.

RizKI T.M. 1978: The circulatory system and associated cells and tissues. In Ashburner M. \& Wright T. (eds): The Genetics and Biology of Drosophila. Vol. 2a. Academic Press, New York, pp. 397-452.

SLÁmA K. 2000: Extracardiac versus cardiac haemocoelic pulsations in pupae of the mealworm (Tenebrio molitor L.). $J$. Insect Physiol. 46: 977-992.

SLÁmA K. 2003: Mechanical aspects of heartbeat reversal in pupae of Manduca sexta. J. Insect Physiol. 49: 645-657.

SLÁMA K. 2004: The effect of Corazonin on heartbeat reversal in pupae of the tobacco hornworm, Manduca sexta. Eur. J. Entomol. 101: 513-521.

SlÁmA K. 2005: Neuropeptides and insect heartbeat: the myth and reality. Pestycydy (Pesticides) 3: 95-107.

SLÁmA K. 2006: Heartbeat reversal after sectioning of the dorsal vessel and removal of the brain in diapausing pupae of Manduca sexta (Lepidoptera: Sphingidae). Eur. J. Entomol. 103: $17-26$.

SlámA K. 2008: Extracardiac haemocoelic pulsations and the autonomic neuroendocrine system (coelopulse) of terrestrial insects. Terrest. Arthrop. Rev. 1: 39-80.

SLÁMA K. \& FARKAŠ R. 2005: Heartbeat patterns during the postembryonic development of Drosophila melanogaster. $J$. Insect Physiol. 51: 489-503.

SlÁmA K. \& Miller T.A. 2001: Physiology of heartbeat reversal in diapausing pupae of the tobacco hornworm, Manduca sexta (Lepidoptera: Sphingidae). Eur. J. Entomol. 98: 415-431.
SLÁMA K. \& RosińSKi G. 2004: Pharmacological effects of proctolin and CCAP on heartbeat in pupae of the tobacco hornworm, Manduca sexta. Physiol. Entomol. 30: 14-28.

SlÁmA K., SAKAI T. \& TAKEDA M. 2006: Effect of corazonin and CCAP on heartbeat in the adult American cockroach (Periplaneta americana). Arch. Insect Biochem. Physiol. 62: 91-103.

TöGEL M. \& PASS G. 2006: Funktionsmorphologische und phylogenetische Aspekte der Herzschlagumkehr bei Insekten. Entomol. Austr. 13: 39-46.

TuBlitZ N. 1989: Insect cardioactive peptides: Neurohormonal regulation of cardiac activity by two cardioacceleratory peptides during flight in the tobacco hawkmoth, Manduca sexta. J. Exp. Biol. 142: 31-48.

TublitZ N. \& Truman J.W. 1985: Insect cardioactive peptides. II. Neurohormonal control of heart activity by two cardioacceleratory peptides in the tobacco hawkmoth, Manduca sexta. J. Exp. Biol. 114: 381-395.

VEENSTRA J.A. 1989: Isolation and structure of corazonin, a cardioactive peptide from American cockroach. FEBS Letters 250: 231-234.

WASSERTHAL L.T. 1996: Interaction of circulation and tracheal ventilation in holometabolous insects. Adv. Insect Physiol. 6: 297-351.

Wasserthal L.T. 1999: Functional morphology of the heart and of a new cephalic pulsatile organ in the blowfly Calliphora vicina (Diptera: Calliphoridae) and their roles in hemolymph transport and tracheal ventilation. Insect Morph. Embryol. 28: 111-129.

Wasserthal L.T. 2007: Drosophila flies combine periodic heartbeat reversal with a circulation in the anterior body mediated by a newly discovered anterior pair of ostial valves and 'venous' channels. J. Exp. Biol. 210: 3707-3719.

Wigglesworth V.B. 1965: The Princioples of Insect Physiology. 6th ed. Methuen, London, New York, 741 pp.

Zeitouni B., Sénatore S., Séverac D., Aknin C., Sémériva M. \& PERrin L. 2007: Signalling pathways involved in adult heart formation revealed by gene expression profiling in Drosophila. PLoS Genetics 3: 1907-1921.

Zornik E., PAisley K. \& Nichols R. 1999: Neural transmitters and a peptide modulate Drosophila heart rate. Peptides 20: $45-51$.

Received March 9, 2009; revised and accepted June 17, 2009 